\title{
Shortening anomalies in supersymmetric theories
}

\author{
Jaume Gomis, ${ }^{a}$ Zohar Komargodski, ${ }^{b}$ Hirosi Ooguri, ${ }^{c, d}$ Nathan Seiberg ${ }^{e}$ and \\ Yifan Wang ${ }^{f}$ \\ ${ }^{a}$ Perimeter Institute for Theoretical Physics, \\ Waterloo, Ontario, N2L 2Y5, Canada \\ ${ }^{b}$ Weizmann Institute of Science, \\ Rehovot 76100, Israel \\ ${ }^{c}$ Walter Burke Institute for Theoretical Physics, \\ Caltech, Pasadena, CA 91125, U.S.A. \\ ${ }^{d}$ Kavli IPMU, WPI, University of Tokyo, \\ Kashiwa, 77-8583, Japan \\ e School of Natural Sciences, Institute for Advanced Study, \\ Princeton, NJ 08540, U.S.A. \\ ${ }^{f}$ Joseph Henry Laboratories, Princeton University, \\ Princeton, NJ 08544, U.S.A. \\ E-mail: jgomis@perimeterinstitute.ca, \\ zohar.komargodski@weizmann.ac.il, ooguri@theory.caltech.edu, \\ seiberg@ias.edu, yifanw@princeton.edu .
}

ABSTRACT: We present new anomalies in two-dimensional $\mathcal{N}=(2,2)$ superconformal theories. They obstruct the shortening conditions of chiral and twisted chiral multiplets at coincident points. This implies that marginal couplings cannot be promoted to background superfields in short representations. Therefore, standard results that follow from $\mathcal{N}=$ $(2,2)$ spurion analysis are invalidated. These anomalies appear only if supersymmetry is enhanced beyond $\mathcal{N}=(2,2)$. These anomalies explain why the conformal manifolds of the $\mathrm{K} 3$ and $T^{4}$ sigma models are not Kähler and do not factorize into chiral and twisted chiral moduli spaces and why there are no $\mathcal{N}=(2,2)$ gauged linear sigma models that cover these conformal manifolds. We also present these results from the point of view of the Riemann curvature of conformal manifolds.

Keywords: Anomalies in Field and String Theories, Conformal Field Theory, Supersymmetric gauge theory

ArXiv EPrint: 1611.03101

Dedicated to John Schwarz on his 75th birthday 


\section{Contents}

1 Introduction 1

2 OPE of chiral and twisted chiral operators in $\mathcal{N}=(2,2)$ SCFT's $\quad 6$

$\begin{array}{lll}3 & \text { Supersymmetric contact terms and the shortening anomaly } & 10\end{array}$

4 Curvature of conformal manifold and factorization $\quad 13$

$5 \mathcal{N}=(4,4)$ conformal manifolds $\quad 15$

$\begin{array}{lll}5.1 & \text { Riemannian curvature } & 16\end{array}$

$\begin{array}{lll}5.2 & \text { Supercurrent bundle } & 17\end{array}$

$\begin{array}{ll}\text { A The Wess-Zumino perspective } & 18\end{array}$

$\begin{array}{ll}\text { B Special geometry relation } & 21\end{array}$

$\begin{array}{ll}\text { C Four-point function involving supercurrents } & 21\end{array}$

$\begin{array}{ll}\text { D SU(2) out selection rules } & 23\end{array}$

\section{Introduction}

Conformal Field Theories (CFT's) often come in a continuous family labeled by their exactly marginal couplings. This family, known as the conformal manifold $\mathcal{M}$, is endowed with a canonical metric, the Zamolodchikov metric [1]. The Zamolodchikov metric is determined by the two-point functions of the exactly marginal operators. The general Riemannian structure of conformal manifolds was first discussed in $[2,3]$.

Such conformal manifolds appear in theories with extended symmetries, such as supersymmetry or current algebra (e.g., the $c=1$ models), and in certain large $N$ theories. $^{1}$ These conformal manifolds also play an important role in the AdS/CFT correspondence and on string worldsheets. In the former case, the conformal manifold of the boundary CFT maps to the space of vacua in the bulk Anti-de Sitter space (AdS). In the latter case, the conformal manifold of the worldsheet theory maps to the space of solutions of the equations of motion in spacetime.

One of the fundamental properties of the conformal manifold of two-dimensional $\mathcal{N}=$ $(2,2)$ superconformal field theories (SCFT's) is that it factorizes locally ${ }^{2}$ into the product

\footnotetext{
${ }^{1}$ Some general arguments about when such families may exist can be found in [4].

${ }^{2}$ There are examples where the conformal manifold is modded out by a discrete symmetry, which prevents it from being a product globally [5, 6]. We thank D. Morrison for a useful discussion about this point.
} 
of two Kähler manifolds,

$$
\mathcal{M}_{c} \times \mathcal{M}_{t c}
$$

Coordinates of $\mathcal{M}_{c}$ and $\mathcal{M}_{t c}$ are the coupling constants of the exactly marginal operators constructed from the dimension $\left(\frac{1}{2}, \frac{1}{2}\right)$ operators in the chiral and twisted chiral rings [7] of the SCFT. When the Virasoro central charge is $c=9$ and the SCFT is realized as a nonlinear sigma model with a Calabi-Yau threefold as its target space, the factorization (1.1) was proven in [8] by combining string theory worldsheet SCFT and target space arguments. ${ }^{3}$ For other early papers on the $\mathcal{N}=(2,2)$ conformal manifold from the target space and the worldsheet view points, see $[9,10]$ and $[8,11-13]$, respectively.

More recently, in [14] the Weyl anomaly on the conformal manifold of $\mathcal{N}=(2,2)$ theories was used to rederive the factorization (1.1). The argument in [14] applies to any $\mathcal{N}=(2,2)$ SCFT, with no restriction on the Virasoro central charge. An assumption made in [14] was that the coupling constants parameterizing $\mathcal{M}_{c}$ and $\mathcal{M}_{t c}$ could be promoted to supersymmetric dimension $(0,0)$ background chiral and twisted chiral multiplets, in the spirit of [15]. Factorization of the conformal manifold (1.1) then followed from the classification of anomalies of the partition function under super-Weyl transformations. Alternatively, one can also easily provide an argument for factorization (1.1) in the spirit of [16].

On the other hand, it is well-known that the conformal manifold of the $n$-dimensional torus $T^{n}$ SCFT is locally [17],

$$
\frac{O(n, n)}{O(n) \times O(n)},
$$

while the conformal manifold of the K3 SCFT is locally [2],

$$
\frac{O(4,20)}{O(4) \times O(20)} .
$$

More generally, the conformal manifold of $\mathcal{N}=(4,4)$ SCFT's is locally of the form [18] (see also [19]),

$$
\frac{O(4, n)}{O(4) \times O(n)},
$$

for some $n .^{4}$ These examples appear to be at odds with the factorization (1.1) of the conformal manifold of $\mathcal{N}=(2,2)$ SCFT's proven in [14]. Indeed, with the exception of the $n=2$ case in (1.2), these conformal manifolds do not factorize locally into a product of Kähler manifolds. In fact, they are not even Kähler manifolds. This is in spite of the fact that the $T^{n}$ SCFT with $n$ even and the other SCFT's enjoy $\mathcal{N}=(2,2)$ superconformal symmetry.

Another way of presenting this puzzle is the following. Normally, when a particular global symmetry of a theory implies some special properties, extending that symmetry does not ruin those properties. Here, we see a counter-example to that. Specifically, if we consider a theory with $\mathcal{N}=(4,4)$ supersymmetry and view it as a special case of a

\footnotetext{
${ }^{3}$ For SCFT's realizing Calabi-Yau compactifications, $\mathcal{M}_{c}$ and $\mathcal{M}_{t c}$ parameterize the moduli space of complex structure and complexified Kähler class.

${ }^{4}$ For a sigma model with a hyper-Kähler target space $M, n=h^{1,1}(M)$.
} 
$\mathcal{N}=(2,2)$ theory, we might conclude that the conformal manifold should be Kähler and that it should factorize as in (1.1). This conclusion turns out to be wrong. Later we will discuss additional properties of $\mathcal{N}=(2,2)$ theories that do not hold true in theories with extended supersymmetry.

In this note we resolve this tension. In a generic $\mathcal{N}=(2,2) \mathrm{SCFT}$, the operator product expansion (OPE) between a chiral multiplet $\mathcal{O}$ and a twisted chiral multiplet $\tilde{\mathcal{O}}$ of scaling dimensions $\left(\frac{1}{2}, \frac{1}{2}\right)$ does not have poles. On the other hand, in all the counter-examples to factorization mentioned above, the OPE has a pole ${ }^{5}$

$$
\mathcal{O}\left|\left(x_{1}\right) \tilde{\mathcal{O}}\right|\left(x_{2}\right) \sim \frac{1}{x_{1}^{--}-x_{2}^{--}} J_{++}\left(x_{2}\right)+\cdots,
$$

where the symbol | on the left-hand side picks up the bottom component of each multiplet. The operator $J_{++}$appearing on the right-hand side must be both chiral and twisted chiral, and with scaling dimensions $(1,0)$ and R-charges $(2,0) . J_{++}$is the bottom component of a special $\mathcal{N}=(2,2)$ short multiplet, which we shall denote by $\mathcal{J}_{++}$. The existence of such a multiplet implies that the R-symmetry is enhanced and that the SCFT enjoys enlarged superconformal symmetry beyond $\mathcal{N}=(2,2)$ supersymmetry. For example, in the K3 SCFT, the current $J_{++}$enlarges the R-symmetry from U(1) to $\mathrm{SU}(2){ }^{6}$

Let us imagine that we deform the SCFT with exactly marginal operators

$$
\int d^{2} x d \theta^{+} d \theta^{-} \lambda \mathcal{O}+\int d^{2} x d \theta^{+} d \bar{\theta}^{-} \tilde{\lambda} \tilde{\mathcal{O}}+c . c
$$

A powerful idea [15] is to promote $\lambda$ and $\tilde{\lambda}$ to background chiral and twisted chiral superfields. We will see that whenever the OPE between $\mathcal{O}$ and $\tilde{\mathcal{O}}$ is singular as in (1.5), then the couplings cannot be promoted to such background superfields due to an anomaly! We can either promote the $\lambda$ s to background chiral superfields or the $\tilde{\lambda}_{\mathrm{s}}$ to background twisted chiral superfields, but we cannot do both simultaneously. To our knowledge, this type of obstruction has not been discussed before.

Before we explain how this comes about let us explain the physics of promoting couplings to background fields. The effective action as a function of the background fields satisfies the required symmetries when the operators do not have contact terms that spoil those symmetries. For example, if a conserved current is not conserved at coincident points then the background effective action will not be gauge invariant. Another example is if in a CFT the operator equation $T_{\mu}^{\mu}=0$ does not hold at coincident points, then the background effective action fails to depend just on the conformal class of the background metric. Similarly, in supersymmetric theories, for the effective action to depend on $\lambda$ and $\tilde{\lambda}$ as chiral and twisted chiral superfields, the operators $\mathcal{O}$ and $\tilde{\mathcal{O}}$ have to obey their defining equations

$$
\bar{D}_{ \pm} \mathcal{O}=0, \quad \bar{D}_{+} \tilde{\mathcal{O}}=D_{-} \tilde{\mathcal{O}}=0
$$

\footnotetext{
${ }^{5}$ Throughout this note we study the theories in Euclidean space. However, we use Lorentzian signature notation with coordinates $x^{ \pm \pm}$etc., which are complex conjugates of each other. The reason for using this notation is that we use complex conjugation notation on the charged chiral objects like the odd coordinates $\theta$, as if we are in Lorentzian signature.

${ }^{6}$ It is important that the current $J_{++}$which enlarges the R-symmetry corresponds to a normalizable state in the SCFT.
} 
not only at separated points (which holds by definition) but also at coincident points. Loosely speaking, one could say that the equations (1.7) have to be obeyed off-shell. As with all anomalies, our shortening anomaly can be understood as the failure of the partition function to be invariant under certain background field transformations. The operator equations (1.7) at coincident points would be a consequence of the partition function being invariant under certain background superfield transformations, akin to the standard way conservation laws follow from the invariance of the partition function under background field transformations. When the partition function is not invariant under these background field transformations, we encounter an anomaly. This point of view is elaborated in appendix $\mathrm{A}$.

We will show that the OPE (1.5) induces contact terms of the form

$$
\begin{aligned}
\bar{D}_{+}^{(1)} \mathcal{O}\left(Z_{1}\right) \tilde{\mathcal{O}}\left(Z_{2}\right) & \sim \delta^{(2)}\left(z_{1 \overline{2}}\right) \theta_{12}^{+} \mathcal{J}_{++}\left(Z_{2}\right) \\
\bar{D}_{+}^{(2)} \mathcal{O}\left(Z_{1}\right) \tilde{\mathcal{O}}\left(Z_{2}\right) & \sim \delta^{(2)}\left(z_{1 \overline{2}}\right) \theta_{12}^{+} \mathcal{J}_{++}\left(Z_{1}\right)
\end{aligned}
$$

and that it is impossible to tune both of these contact terms away (the notation will be explained in section 3.) Therefore, the effective action does not depend on the background coupling constants as if they were chiral and twisted chiral superfields. Some of the background couplings have to sit in long multiplets. We call this phenomenon a shortening anomaly. ${ }^{7}$ The discussion above is reminiscent of the clash between conservation of the vector and axial current in theories with fermions, where by adjusting counter-terms either symmetry can be preserved, but not both simultaneously.

As is standard in such situations, where some operator equations are violated at coincident points, when we turn on nontrivial backgrounds for $\lambda$ and $\tilde{\lambda}$ then the contact terms (1.8) lead to nontrivial operator equations. Depending on which counter-terms we choose, one of the equations below has to be true:

$$
\begin{aligned}
& \bar{D}_{+} \mathcal{O} \sim \bar{D}_{-} \tilde{\lambda} \mathcal{J}_{++}, \\
& \bar{D}_{+} \tilde{\mathcal{O}} \sim D_{-} \lambda \mathcal{J}_{++} .
\end{aligned}
$$

For constant couplings, where only the bottom components of $\lambda$ and $\tilde{\lambda}$ are turned on, the operators $\mathcal{O}$ and $\tilde{\mathcal{O}}$ remain short, as they should.

The obstruction to promoting both chiral and twisted chiral couplings to short multiplets (which exists only if supersymmetry is enhanced) invalidates the conclusions found in [14] for such theories. ${ }^{8}$ We therefore conclude that factorization (1.1) breaks down only if the supersymmetry algebra is larger than $\mathcal{N}=(2,2)$. The SCFT's with conformal man-

\footnotetext{
${ }^{7}$ We would like to emphasize that the operators $\mathcal{O}, \tilde{\mathcal{O}}$ remain short in the standard situation where the couplings are constant. Indeed, $\lambda, \tilde{\lambda}$ have vanishing beta functions, since the operators $\mathcal{O}, \tilde{\mathcal{O}}$ have no operators to combine with (see [20] and also earlier literature, e.g. [11]). In particular, (1.5) does not induce a beta function. The shortening anomaly is in the background fields, not in the operators. The operator equations are modified only in nontrivial configurations for the background superfields (see equation (1.9)).

${ }^{8}$ The analysis in the spirit of [16] is also invalidated by this anomaly since an implicit assumption in such an analysis is that it is possible to preserve the shortening conditions of all the dimension $\left(\frac{1}{2}, \frac{1}{2}\right)$ chiral and twisted chiral operators both at separate and coincident points.
} 
ifolds (1.2) (with $n>2$ even), (1.3) and (1.4) are indeed endowed with such an operator of R-charge $(2,0)$ and have enhanced supersymmetries, thus resolving the paradox. ${ }^{9}$

This phenomenon is similar to a familiar situation in supergravity. The target space of $\mathcal{N}=1$ supergravity in $4 d$ is known to be Kähler (see [21] for details). But $\mathcal{N}=2$ supergravity with hypermultiplets has a quaternionic target space, which is not Kähler (see [22] for a review of the scalar manifolds of supergravities in various dimensions). There is no contradiction in that because the $\mathcal{N}=2$ theory is not merely a special $\mathcal{N}=1$ supergravity theory because it has a multiplet including a graviphoton and a gravitino, which is not present in the generic $\mathcal{N}=1$ theory. The special $\mathcal{N}=(2,2)$ multiplet $\mathcal{J}_{++}$ that resolves our puzzle, which includes a conserved spin- $\frac{3}{2}$ current, plays a similar role to the graviphoton multiplet in this supergravity analog. For a related discussion see [23].

We can relate our discussion to supergravity more directly if we view our $2 d$ models as worldsheet theories for strings and we study their spacetime description. The low energy description of string compactifications on Calabi-Yau threefolds, described by $\mathcal{N}=(2,2)$ SCFT's on the worldsheet, is captured by four-dimensional $\mathcal{N}=2$ supergravity, whose scalar manifold indeed takes the factorized form (1.1). On the other hand, compactifications on manifolds leading to enhanced spacetime supersymmetry, such as $T^{6}$ or $\mathrm{K} 3 \times T^{2}$, are instead described by $\mathcal{N}=8$ or $\mathcal{N}=4$ supergravity. The scalar manifold in these theories does not factorize. ${ }^{10}$

As with all 't Hooft-like anomalies, our analysis leads to theorems about the nonexistence of certain renormalization group (RG) flows. If there exists a strictly $\mathcal{N}=(2,2)$ supersymmetric RG flow in which all the infrared marginal couplings are realized as chiral or twisted chiral couplings along the flow, then it is guaranteed that the corresponding coupling constants in the infrared are in short representations and hence there is no shortening anomaly. Therefore, the conformal manifold would have to factorize into a chiral Kähler manifold and a twisted chiral Kähler manifold. Therefore, one can immediately conclude that there cannot exist an $\mathcal{N}=(2,2)$ RG flow that realizes the full conformal manifold of the K3 SCFT. Indeed, constructions of gauged linear sigma models (GLSM's) [24] which lead to subspaces of the K3 conformal manifold are known (see for example [18, 25]), but it has never been possible to embed the K3 SCFT in a UV completion that covers the full conformal manifold. The same holds for $T^{4}$ but not for $T^{2}$, which does not have a shortening anomaly, and indeed admits an $\mathcal{N}=(2,2)$ GLSM representation that realizes the complete $T^{2}$ SCFT conformal manifold. ${ }^{11}$ We now see that the obstruction for K3 and $T^{4}$ is due to an anomaly in the infrared that must be matched in the ultraviolet. ${ }^{12}$

\footnotetext{
${ }^{9}$ Such an operator does not exist for the $T^{2}$ SCFT. And indeed, the $T^{2}$ conformal manifold (1.2) factorizes into a product of Kähler manifolds, locally given by $\mathrm{SL}(2) / \mathrm{U}(1) \times \mathrm{SL}(2) / \mathrm{U}(1)$.

${ }^{10}$ The conformal manifold of the worldsheet SCFT is a subspace of the supergravity scalar manifold.

${ }^{11}$ The GLSM is an $\mathcal{N}=(2,2) \mathrm{U}(1)$ gauge theory with chiral multiplets $\left(X_{1}, X_{2}, X_{3}, P\right)$ of charges $(1,1,1,-3)$ with a superpotential $W=P G_{3}(X)$, where $G_{3}(X)$ is a homogeneous polynomial of degree 3. The twisted chiral coupling is realized by the complexified FI parameter and the chiral coupling by the single complex parameter in $G_{3}(X)$.

${ }^{12}$ Our analysis however does not rule out $\mathcal{N}=(4,4)$ supersymmetric RG flows that would cover the full conformal manifold of sigma models on K3 or $T^{4}$.
} 
It is interesting to relate this discussion to recent developments concerning the $S^{2}$ partition functions of $\mathcal{N}=(2,2)$ SCFT's [26-28]-[29]. There are two inequivalent ways to compactify such theories on $S^{2}$ and they compute, respectively, the Kähler potentials of the chiral and twisted chiral deformations [28, 30] (see also [14, 31]). However, this statement is not meaningful, if the total space does not factorize as in (1.1)! Therefore, when our anomaly is present, also this result about general $\mathcal{N}=(2,2)$ SCFT's is invalidated. (One can understand it again as being due to the failure of spurion analysis.) Because of the anomaly, there is no $\mathcal{N}=(2,2)$ UV completion that would cover the full conformal manifold, therefore, what one can extract from the sphere partition function is at best the Kähler potential on some Kähler submanifolds of the conformal manifold. An alternative sphere compactification that utilizes the extended supersymmetry may exist and it may probe the full conformal manifold.

We also provide a complementary perspective on the anomalies by studying the Riemann curvature of the conformal manifold in $\mathcal{N}=(2,2)$ SCFT's, extending the previous work $[8,19,32]$. The study of the mixed chiral and twisted chiral exactly marginal curvature components leads us to establish a factorization theorem: the conformal manifold of an $\mathcal{N}=(2,2)$ SCFT fails to factorize, if and only if the SCFT is endowed with a conserved current of R-charges $(2,0)$, precisely the same operator $J_{++}$responsible for the shortening anomaly.

The outline of the paper is as follows. In section 2 we set the stage by discussing the OPE of chiral and twisted chiral superfields. In section 3 we show that if a particular short representation $\mathcal{J}_{++}$appears in the OPE, then one inevitably finds an obstruction to imposing the shortening conditions simultaneously on both the chiral and twisted chiral superfields. In section 4 the same result is established by analyzing the Riemann curvature tensor of the conformal manifold of $\mathcal{N}=(2,2)$ SCFT's. In section 5 we study the curvature of the conformal manifold of SCFT's endowed with the small $\mathcal{N}=(4,4)$ superconformal algebra and prove that the conformal manifold of such theories indeed takes the form (1.4). In this section we further show that the extended supercharges acquire non-trivial holonomies when transported around the conformal manifold.

In appendix A, we discuss the shortening anomaly from the Wess-Zumino point of view. Some of the calculations of the various curvatures are presented in appendices B, C, and D.

\section{OPE of chiral and twisted chiral operators in $\mathcal{N}=(2,2)$ SCFT's}

Around a given point on the conformal manifold of an $\mathcal{N}=(2,2)$ SCFT an exactly marginal operator can be realized as the top component of a chiral multiplet $\mathcal{O}$ with $\mathrm{U}(1)_{+} \times \mathrm{U}(1)_{-}$ R-charges $(1,1)$ or as the top component of a twisted chiral multiplet $\tilde{\mathcal{O}}$ with R-charges $(1,-1)$. These multiplets obey the shortening conditions

$$
\begin{array}{rlrl}
\bar{D}_{+} \mathcal{O} & =0 & & \bar{D}_{-} \mathcal{O}=0 \\
\bar{D}_{+} \tilde{\mathcal{O}}=0 & D_{-} \tilde{\mathcal{O}}=0 .
\end{array}
$$


Our aim is to determine to what extent these shortening conditions can be maintained as we explore the conformal manifold of an $\mathcal{N}=(2,2)$ SCFT.

Monitoring the shortening conditions (2.1) under an exactly marginal perturbation

$$
\int d^{2} x d \theta^{+} d \theta^{-} \lambda \mathcal{O}+\int d^{2} x d \theta^{+} d \bar{\theta}^{-} \tilde{\lambda} \tilde{\mathcal{O}}+c . c
$$

leads us to analyze the contact terms in

$$
\left[\bar{D}_{ \pm} \mathcal{O}\left(Z_{1}\right)\right] \tilde{\mathcal{O}}\left(Z_{2}\right), \quad \mathcal{O}\left(Z_{1}\right)\left[\bar{D}_{+} \tilde{\mathcal{O}}\left(Z_{2}\right)\right], \quad \mathcal{O}\left(Z_{1}\right)\left[D_{-} \tilde{\mathcal{O}}\left(Z_{2}\right)\right]
$$

where $Z_{I} \equiv\left(x_{I}^{ \pm \pm}, \theta_{I}^{ \pm}, \bar{\theta}_{I}^{ \pm}\right)$are points in superspace. In this section we determine the operator product expansion (OPE) of a chiral and a twisted chiral multiplets, whose top components yield marginal operators, leaving the analysis of contact terms to the following section.

In supersymmetry, it is often useful to employ spurion analysis. E.g., the coupling constants of chiral operators $\mathcal{O}$ are promoted to background chiral multiplets [15] . This procedure makes sense only if the operator equation $\bar{D}_{ \pm} \mathcal{O}=0$ is respected also at coincident points (loosely speaking, we can say that $\mathcal{O}$ is chiral off-shell). This is because when we write the partition function depending on some background fields, by taking derivatives with respect to the background fields, we can probe the correlation functions of the corresponding operators both at separated and at coincident points. By definition, operators equations are always obeyed at separated points, but they may fail at coincident points. The famous (continuous) 't Hooft anomalies arise when a conservation equation is not obeyed at coincident points. As a consequence, the partition function depending on the associated background fields does not obey the naively expected equations (in the famous case of the chiral anomaly, the partition function is not gauge invariant). Similarly, we can couple twisted chiral background fields to twisted chiral operators as long as the corresponding shortening conditions are valid off-shell.

A general method that guarantees that some conservation equation is obeyed also at coincident points is to construct a regularization obeying the conservation equation. This automatically tunes the contact terms in the infrared to zero. Such a regularization may not exist, if there is a genuine anomaly in the conservation equation. In the next section we will establish the existence of some contact terms that violate the shortening conditions (2.1) at coincident points. Therefore, the standard arguments relying on the selection rules of background superfields are not valid. This also means that, when this occurs, $\mathcal{N}=(2,2)$ RG flows that contain all the infrared chiral and twisted chiral couplings cannot exist.

We will show that such a subtlety in the spurion analysis takes place in an $\mathcal{N}=(2,2)$ SCFT in two dimensions when it has an operator of dimensions $(1,0)$ and R-charges $(2,0)$. Unitarity implies that this operator, which we denote by $J_{++}$, is a conserved current, obeying $\partial_{--} J_{++}=0$. Since $J_{++}$carries a non-vanishing R-charge, its existence implies that the supersymmetry is enhanced.

A typical (but not the only) example of supersymmetry enhanced by such an extra R-current is the small $\mathcal{N}=4$ superconformal symmetry with $\mathrm{SU}(2)_{R}$ symmetry. In an 
$\mathcal{N}=(4,4)$ SCFT, chiral and twisted chiral operators are rotated into each other by the extra R-current as

$$
J_{++}\left(x_{1}\right) \overline{\tilde{\mathcal{O}}}\left|\left(x_{2}\right) \sim \frac{1}{x_{1}^{++}-x_{2}^{++}} \mathcal{O}\right|\left(x_{2}\right),
$$

where $\mathcal{O} \mid$ denotes the bottom component of the operator $\mathcal{O}$. By taking OPE's of both sides of the equation with another twisted chiral operator $\tilde{\mathcal{O}}\left(x_{3}\right)$ (or, in other words, using the symmetry of the OPE coefficients), we find

$$
\mathcal{O}\left|\left(x_{2}\right) \tilde{\mathcal{O}}\right|\left(x_{3}\right) \sim \frac{1}{x_{2}^{--}-x_{3}^{--}} J_{++}\left(x_{3}\right)+\cdots,
$$

where $\cdots$ encode the contributions of non-chiral operators and descendants. For simplicity, we are suppressing some coefficients that we will make more explicit later. In particular, when the $\mathcal{N}=(4,4)$ SCFT is realized as a non-linear sigma model whose target space is a hyper-Kähler manifold $M$, there is a unique holomorphic 2-form $\Omega \in H^{2,0}(M)$, and the extra R-current is expressed as $J_{++}=\Omega_{a b} \psi_{+}^{a} \psi_{+}^{b}$. Chiral and twisted chiral operators are expressed as,

$$
\mathcal{O}\left|=k_{a \bar{b}} \psi_{+}^{a} \bar{\psi}_{-}^{\bar{b}}, \quad \tilde{\mathcal{O}}\right|=k_{a \bar{b}} g^{\bar{b} c} \Omega_{c d} \psi_{+}^{a} \psi_{-}^{d},
$$

where $k \in H^{1,1}(M), g_{a \bar{b}}$ is the Kähler metric on $M$, and $\psi_{ \pm}$are fermions. The OPE (2.5) then follows from $\psi_{-}^{\bar{b}}\left(x_{3}\right) \psi_{-}^{d}\left(x_{2}\right) \sim \frac{g^{\bar{b} d}}{x_{3}^{--}-x_{2}^{--}}$.

More generally, one can show that the existence of $J_{++}$of dimensions $(1,0)$ and Rcharges $(2,0)$ alone is sufficient for the pairing (2.4) and therefore the OPE (2.5) follows without assuming the small $\mathcal{N}=4$ superconformal symmetry. Indeed, since $J_{++}$does not commute with the $\mathrm{U}(1)_{+} \times \mathrm{U}(1)_{-}$R-symmetry in an $\mathcal{N}=(2,2)$ SCFT, the R-symmetry group must be larger than just $\mathrm{U}(1)_{+} \times \mathrm{U}(1)_{-}$and the dimension $\left(\frac{1}{2}, \frac{1}{2}\right)$ chiral and twisted chiral operators must furnish a representation of it. Therefore, $J_{++}$cannot act trivially and hence some pairing as in (2.4) and (2.5) must be present.

Unitarity and supersymmetry further imply that the extra R-current $J_{++}$is the bottom component of a very short multiplet, which we denote by $\mathcal{J}_{++}$. It obeys

$$
\bar{D}_{+} \mathcal{J}_{++}=\bar{D}_{-} \mathcal{J}_{++}=D_{-} \mathcal{J}_{++}=0 .
$$

For convenience, we summarize the R-charges of various objects used in the following:

\begin{tabular}{ccc}
\multicolumn{4}{c}{$\mathrm{U}(1)_{+}$} & $\mathrm{U}(1)_{-}$ \\
$\theta^{+}$ & 1 & 0 \\
$\theta^{-}$ & 0 & 1 \\
$D_{+}$ & -1 & 0 \\
$D_{-}$ & 0 & -1 \\
$\mathcal{O}$ & 1 & 1 \\
$\tilde{\mathcal{O}}$ & 1 & -1 \\
$\mathcal{J}_{++}$ & 2 & 0 \\
$\mathcal{J}_{--}$ & 0 & 2
\end{tabular}

Our final goal is to monitor the shortening conditions (2.1) as we explore the conformal manifold while preserving $\mathcal{N}=(2,2)$ supersymmetry. This prompts us to determine 
the OPE of the supermultiplets $\mathcal{O}, \tilde{\mathcal{O}}$ and $\mathcal{J}_{++}$in $\mathcal{N}=(2,2)$ superspace, which makes supersymmetry manifest. Introducing coordinates $(x, \theta, \bar{\theta})$ (suppressing the,+- indices) we define

$$
D=\frac{\partial}{\partial \theta}-i \bar{\theta} \partial, \quad \bar{D}=-\frac{\partial}{\partial \bar{\theta}}+i \theta \partial
$$

which generate

$$
\{D, \bar{D}\}=2 i \partial \text {. }
$$

The chiral coordinates $y \equiv x-i \theta \bar{\theta}$ and $\bar{y} \equiv x+i \theta \bar{\theta}$ obey

$$
\bar{D} y=0 \quad D \bar{y}=0
$$

and are chiral/anti-chiral respectively, which we denote also as $c / \bar{c}$. In this language $\mathcal{O}$ is $(c, c), \tilde{\mathcal{O}}$ is $(c, \bar{c})$ and $\mathcal{J}_{++}$is simultaneously $(c, c)$ and $(c, \bar{c})$.

We want to determine the dependence of three-point correlators on the superspace position of the operators. We start with the supertranslation Ward identities. Given two points in superspace $\left(x_{1}, \theta_{1}, \bar{\theta}_{1}\right)$ and $\left(x_{2}, \theta_{2}, \bar{\theta}_{2}\right)$, we can define two independent even linear invariants: ${ }^{13}$

$$
\begin{aligned}
& z_{1 \overline{2}} \equiv x_{1}-x_{2}-i \theta_{2} \bar{\theta}_{1}+i \theta_{1} \bar{\theta}_{2}-i \theta_{12} \bar{\theta}_{12}=y_{1}-\bar{y}_{2}+2 i \theta_{1} \bar{\theta}_{2} \\
& z_{\overline{1} 2} \equiv x_{1}-x_{2}+i \theta_{1} \bar{\theta}_{1}+i \theta_{2} \bar{\theta}_{2}-2 i \theta_{2} \bar{\theta}_{1}=\bar{y}_{1}-y_{2}-2 i \theta_{2} \bar{\theta}_{1}
\end{aligned} .
$$

Supertranslation invariance implies that correlators depend on the position of operators through $z_{i \bar{j}}$ and $z_{\bar{i} j}$. The coordinates we have defined are rather convenient. Indeed, if the $i$-th operator is chiral, the correlators depend on $z_{i \bar{j}}$ only, while if it is anti-chiral they depend on $z_{\bar{i} j}$.

Our correlator of interest is:

$$
\left\langle\mathcal{O}\left(Z_{1}\right) \tilde{\mathcal{O}}\left(Z_{2}\right) \overline{\mathcal{J}}_{++}\left(Z_{3}\right)\right\rangle
$$

where $Z_{I} \equiv\left(x_{I}^{ \pm \pm}, \theta_{I}^{ \pm}, \bar{\theta}_{I}^{ \pm}\right)$. The shortening conditions,

$$
\begin{aligned}
\bar{D}_{+} \mathcal{O} & =\bar{D}_{-} \mathcal{O}=0 \\
\bar{D}_{+} \tilde{\mathcal{O}} & =D_{-} \tilde{\mathcal{O}}=0 \\
D_{+} \overline{\mathcal{J}}_{++} & =\bar{D}_{-} \overline{\mathcal{J}}_{++}=D_{-} \overline{\mathcal{J}}_{++}=0
\end{aligned}
$$

together with supertranslational and rotational invariance imply that the correlator (2.13) depends on $z_{1 \overline{3}}^{++}, z_{2}^{++}$and $z_{1 \overline{2}}^{--}$. A subtlety in SCFT's that must be taken into account is the existence of a superconformal invariant $X\left(Z_{1}, Z_{2}, Z_{3}\right)$ constructed out of three points in superspace [33]. Superconformal invariance and nilpotency of $X\left(Z_{1}, Z_{2}, Z_{3}\right)$ imply that the most general correlator consistent with superconformal invariance is given by

$$
\left\langle\mathcal{O}\left(Z_{1}\right) \tilde{\mathcal{O}}\left(Z_{2}\right) \overline{\mathcal{J}}_{++}\left(Z_{3}\right)\right\rangle=\frac{C}{z_{1 \overline{3}}^{++} z_{2 \overline{3}}^{++} z_{1 \overline{2}}^{--}}\left[1+a X\left(Z_{1}, Z_{2}, Z_{3}\right)\right]
$$

\footnotetext{
${ }^{13}$ The canonical invariants are constructed from the Maurer Cartan one-form $g^{-1} d g=(d x-i \theta d \bar{\theta}+$ $i d \theta \bar{\theta}, d \theta, d \bar{\theta})$, where $g=\exp (-i(x P+\theta Q+\bar{\theta} \bar{Q}))$. This yields the supertranslation invariants $\left(\Delta_{12} \equiv\right.$ $\left.x_{1}-x_{2}-i \theta_{2} \bar{\theta}_{1}+i \theta_{1} \bar{\theta}_{2}, \theta_{12} \equiv \theta_{1}-\theta_{2}, \bar{\theta}_{12} \equiv \bar{\theta}_{1}-\bar{\theta}_{2}\right)$. Note that $z_{1 \overline{2}}=\Delta_{12}-i \theta_{12} \bar{\theta}_{12}$ and $z_{\overline{1} 2}=\Delta_{12}+i \theta_{12} \bar{\theta}_{12}$.
} 
Imposing that $\mathcal{O}\left(Z_{1}\right)$ is chiral forces $a=0$, as $X\left(Z_{1}, Z_{2}, Z_{3}\right)$ depends on $\bar{\theta}_{1}$ [33]. We therefore conclude that the correlator at separated points is

$$
\left\langle\mathcal{O}\left(Z_{1}\right) \tilde{\mathcal{O}}\left(Z_{2}\right) \overline{\mathcal{J}}_{++}\left(Z_{3}\right)\right\rangle=\frac{C}{z_{1 \overline{3}}^{++} z_{2 \overline{3}}^{++} z_{1 \overline{2}}^{--}} .
$$

Moreover, using that

$$
\left\langle\mathcal{J}_{++}\left(Z_{2}\right) \overline{\mathcal{J}}_{++}\left(Z_{3}\right)\right\rangle=\left(\frac{1}{z_{2 \overline{3}}^{++}}\right)^{2}
$$

we obtain our desired OPE:

$$
\mathcal{O}\left(Z_{1}\right) \tilde{\mathcal{O}}\left(Z_{2}\right) \sim \frac{C}{z_{1 \overline{2}}^{--}} \mathcal{J}_{++}\left(Z_{2}\right) .
$$

The superspace correlators we have constructed obey, by construction, the shortening conditions (2.14) at separated points. Our next task is to study the shortening conditions at coincident points (2.3) and determine whether counter-terms can be adjusted so that the shortening conditions for chirals and twisted chirals (2.1) can be both simultaneously obeyed.

\section{Supersymmetric contact terms and the shortening anomaly}

The OPE (2.18) may lead to some contact terms (2.3). ${ }^{14}$ To understand these we need to study the superspace derivatives (the derivatives with respect to the second argument follow from these)

$$
D_{+}^{(1)}\left(\frac{1}{z_{1 \overline{2}}^{--}}\right), \quad \bar{D}_{+}^{(1)}\left(\frac{1}{z_{1 \overline{2}}^{--}}\right) .
$$

Our strategy to compute (3.1) is to extend the well-known formula, $\partial_{++}\left(\frac{1}{x_{12}^{--}}\right)=\pi \delta^{(2)}\left(x_{12}\right)$, i.e.,

$$
\partial_{++}\left(\frac{1}{x_{12}^{--}}\right)=\partial_{++} \partial_{--} \log \left(\left|x_{12}\right|^{2}\right)=\frac{1}{4} \square \log \left(\left|x_{12}\right|^{2}\right)=\pi \delta^{(2)}\left(x_{12}\right),
$$

to Green's function in superspace. This line of inquiry makes manifest an inherent ambiguity in defining the derivatives (3.1) in superspace. In order to define these derivatives we need to specify the behavior of Green's function in superspace for the left-movers, and different choices yield different answers. This ambiguity is a manifestation of the fact that the pole $1 / z_{1 \overline{2}}^{--}$in $\mathcal{N}=(2,2)$ superspace is too singular to yield an unambiguous distribution. ${ }^{15}$ In fact, we will find an ambiguity of $\theta_{12}^{+} \bar{\theta}_{12}^{+} \delta^{(2)}\left(z_{1 \overline{2}}\right)$ in defining $1 / z_{1 \overline{2}}^{-\overline{2}}$. This is analogous to the well-known ambiguity in the distribution $1 / x$ on the real line $x \in R$ by

\footnotetext{
${ }^{14} \mathrm{~A}$ related discussion appeared in [34], where it clarified the need for a contact term, which had been found earlier in [35, 36]. A more modern discussion of that problem appeared in [37].

${ }^{15}$ On the other hand, the pole $1 / x^{--}$on the 2-plane of $\left(x^{++}, x^{--}\right)$can be extended to an unambiguous distribution because $\int d^{2} x f\left(x^{++}, x^{--}\right) / x^{--}$is well-defined for any smooth function $f$. The pole $1 / z_{1 \overline{2}}^{--}$in superspace, however, is akin to $1 /\left(x^{--}\right)^{2}$ and cannot be extended to an unambiguous distribution. The distribution defined via $1 /\left(x^{--}\right)^{2} \equiv-\partial_{--}\left(1 / x^{--}\right)$corresponds to a particular choice of regularization.
} 
the addition of $\delta(x) .{ }^{16}$ As a result, the derivatives (3.1) suffer from certain ambiguities. However, we will find that, whichever choice we make for these ambiguities, we cannot remove the contact terms for both chiral and twisted chiral operators simultaneously.

We can compute (3.1) starting from the Green's functions in superspace

$$
D_{+}^{(1)} D_{-}^{(1)} \log \left(z_{1 \overline{2}}^{++} z_{1 \overline{2}}^{--}\right)=-4 \pi \delta^{(2)}\left(z_{1 \overline{2}}\right) \bar{\theta}_{12}^{+} \bar{\theta}_{12}^{-}
$$

or alternatively

$$
\bar{D}_{+}^{(1)} D_{-}^{(1)} \log \left(z_{\overline{1} 2}^{++} z_{1 \overline{2}}^{--}\right)=4 \pi \delta^{(2)}\left(z_{1 \overline{2}}\right) \theta_{12}^{+} \bar{\theta}_{12}^{-} .
$$

Physically, (3.3) and (3.4) can be interpreted as the Schwinger-Dyson equations or the Ward identities for the shift symmetry of a chiral multiplet and a twisted chiral multiplet respectively. But these are not the only choices. Consider the following identity

$$
\left(\frac{1}{z_{1 \overline{2}}^{--}}\right) D_{-}^{(1)} z_{1 \overline{2}}^{--}=D_{-}^{(1)}\left[a \log \left(z_{1 \overline{2}}^{++} z_{1 \overline{2}}^{--}\right)+b \log \left(z_{\overline{1} 2}^{++} z_{1 \overline{2}}^{--}\right)\right],
$$

which holds as long as $a+b=1$. Using equations (3.3) and (3.4) we arrive at ${ }^{17}$

$$
\begin{aligned}
& D_{+}^{(1)}\left(\frac{1}{z_{1 \overline{2}}^{-}}\right)=-2 \pi i a \delta^{(2)}\left(z_{1 \overline{2}}\right) \bar{\theta}_{12}^{+}, \\
& \bar{D}_{+}^{(1)}\left(\frac{1}{z_{1 \overline{2}}^{--}}\right)=2 \pi i b \delta^{(2)}\left(z_{1 \overline{2}}\right) \theta_{12}^{+} .
\end{aligned}
$$

These expressions obey the supersymmetry algebra for all $a+b=1$

$$
\left\{D_{+}, \bar{D}_{+}\right\}=2 i \partial_{++} .
$$

Amongst these, there are distinguished canonical choices

$$
\begin{aligned}
& D_{+}^{(1)}\left(\frac{1}{z_{1 \overline{2}}^{--}}\right) \bar{D}_{+}^{(1)}\left(\frac{1}{z_{1 \overline{2}}^{--}}\right) \\
& \text {Preserves chiral Ward identity } \quad-2 \pi \quad 0 \\
& \text { Preserves twisted chiral Ward identity } \quad 0 \quad 2 \pi \\
& \text { Symmetric violation of Ward identities }-\pi \quad \pi
\end{aligned}
$$

We note that there is no choice of $a$ that preserves simultaneously the chiral and twisted chiral Ward identity. Even though the derivatives are subject to some ambiguities, we now proceed to unambiguously establish our shortening anomaly.

\footnotetext{
${ }^{16} 1 / x$ is characterized by the fact that $x^{n} \cdot 1 / x=x^{n-1}$ for all positive integers $n$. Therefore, any ambiguity in $1 / x$ must be annihilated by multiplication by $x^{n}$, and the only distribution with this property is proportional to $\delta(x)$ (See, for example, theorem 9 in [38]). Indeed, the principal value $P(1 / x)$ and $1 /(x \pm i \epsilon)$ differ by distributions proportional to $\delta(x)$.

${ }^{17}$ Note that equations (3.3) and (3.4) only fix $D_{+}^{(1)}\left(1 / z_{1 \overline{2}}^{--}\right)$and $\bar{D}_{+}^{(1)}\left(1 / z_{1 \overline{2}}^{--}\right)$up to terms proportional to $\bar{\theta}_{12}^{-}$. However such terms are forbidden by the condition that $\bar{D}_{-}^{(1)}$ annihilates $D_{+}^{(1)}\left(1 / z_{1 \overline{2}}^{--}\right)$ and $\bar{D}_{+}^{(1)}\left(1 / z_{1 \overline{2}}^{--}\right)$.
} 
Anomalies arise when we have an operator that should satisfy an operator equation, such as (2.1), and one finds that such an equation is only correct at separated points while at coincident points there are various contact terms. ${ }^{18}$ Establishing the anomaly amounts to showing that these contact terms cannot be removed by redefining the scheme since scheme redefinitions change the theory by contact terms.

We consider $\mathcal{N}=(2,2)$ supersymmetric contact terms since we assume that the conformal manifold can be explored while maintaining $\mathcal{N}=(2,2)$ supersymmetry. Therefore, the most general OPE between a chiral and a twisted chiral superfield in a theory with an enhanced R-symmetry, now allowing for supersymmetric contact terms, is given by

$$
\mathcal{O}\left(Z_{1}\right) \tilde{\mathcal{O}}\left(Z_{2}\right) \sim \frac{C}{z_{1 \overline{2}}^{--}} \mathcal{J}_{++}\left(Z_{2}\right)+r \delta^{(2)}\left(z_{1 \overline{2}}\right) \bar{\theta}_{12}^{+} \theta_{12}^{+} \mathcal{J}_{++}\left(Z_{2}\right)
$$

where $r$ is a scheme dependent constant, which can be shifted by changing the scheme.

In order to establish our shortening anomaly we must show that it is not possible to tune the contact term $r$ such that the shortening conditions for a chiral and a twisted chiral multiplet can be maintained simultaneously. Since the contact term in (3.9) is annihilated by $\bar{D}_{-}^{(1)}$ and $D_{-}^{(2)}$, these shortening equations are automatically preserved. Acting with $\bar{D}_{+}^{(1)}$ and $\bar{D}_{+}^{(2)}$ we get using $(3.6)^{19}$

$$
\begin{aligned}
& \bar{D}_{+}^{(1)} \mathcal{O}\left(Z_{1}\right) \tilde{\mathcal{O}}\left(Z_{2}\right) \sim 2 \pi i C b \delta^{(2)}\left(z_{1 \overline{2}}\right) \theta_{12}^{+} \mathcal{J}_{++}\left(Z_{2}\right)-r \delta^{(2)}\left(z_{1 \overline{2}}\right) \theta_{12}^{+} \mathcal{J}_{++}\left(Z_{2}\right), \\
& \bar{D}_{+}^{(2)} \mathcal{O}\left(Z_{1}\right) \tilde{\mathcal{O}}\left(Z_{2}\right) \sim 2 \pi i C a \delta^{(2)}\left(z_{1 \overline{2}}\right) \theta_{12}^{+} \mathcal{J}_{++}\left(Z_{1}\right)+r \delta^{(2)}\left(z_{1 \overline{2}}\right) \theta_{12}^{+} \mathcal{J}_{++}\left(Z_{1}\right) .
\end{aligned}
$$

Preserving the chiral and twisted chiral shortening conditions along the conformal manifold requires tuning the coefficient of the supersymmetric counter-term to obey

$$
\begin{aligned}
\text { chiral : } & & r=2 \pi i C b, \\
\text { twisted chiral : } & & r=-2 \pi i C a .
\end{aligned}
$$

However, since $a+b=1$, it is impossible to solve both equations. This implies that we cannot simultaneously preserve the chiral and twisted chiral shortening conditions along the conformal manifold. By tuning the contact term, we can either preserve the chiral or the twisted chiral constraint, but not both. This is our shortening anomaly.

The fact that we cannot preserve both constraints simultaneously is analogous to the situation in two dimensions with vector and axial anomalies [39], where contact terms cannot remove both anomalies simultaneously. For a complementary derivation of our shortening anomaly in the cohomological approach based on the Wess-Zumino consistency conditions, see appendix A.

The contact terms we encountered lead to an operatorial violation of the shortening equations upon deforming the theory by background superfield sources for the exactly

\footnotetext{
${ }^{18} \mathrm{By}$ turning on suitable background fields the ambiguities in the contact terms can be described as an ambiguity in adding local counter-terms constructed out of the operators in the theory and the background fields. When such background fields are present, the problem with contact terms can be uplifted to problems at separated points.

${ }^{19}$ The formula $\bar{D}_{+}^{(2)} \frac{1}{z_{1 \overline{2}}^{-}-}=2 \pi i a \delta^{(2)}\left(z_{1 \overline{2}}\right) \theta_{12}^{+}$follows from $(3.6)$.
} 
marginal operators (as in (2.2)). The shortening conditions become

$$
\begin{aligned}
& \bar{D}_{+} \mathcal{O} \sim \bar{D}_{-} \tilde{\lambda} \mathcal{J}_{++}, \\
& \bar{D}_{+} \tilde{\mathcal{O}} \sim D_{-} \lambda \mathcal{J}_{++} .
\end{aligned}
$$

This implies that promoting the couplings of dimension $\left(\frac{1}{2}, \frac{1}{2}\right)$ chiral and twisted chiral operators to full-fledged background chiral and twisted chiral superfields is impossible whenever the theory includes the operator $J_{++}$. We emphasize that the violations in (3.12) depend on the fermionic components of the multiplet to which the couplings have been promoted.

\section{Curvature of conformal manifold and factorization}

A CFT with exactly marginal operators comes equipped with additional structure beyond the metric on the conformal manifold $\mathcal{M}$. Operators in the CFT are sections of vector bundles over $\mathcal{M}$. A canonical example of this is the set of exactly marginal operators, which are sections of the tangent bundle $T \mathcal{M}$. Transporting operators in the vector bundle along $\mathcal{M}$ leads to operator mixing, which is governed by a connection on the vector bundle $[3,40,41]$. The curvature of these connections captures geometrical and topological data about the vector bundle of operators in the CFT.

The curvature of the tangent bundle $T \mathcal{M}$ can be used to prove theorems about the conformal manifold $\mathcal{M}$. The computation in [8] of the curvature of $T \mathcal{M}$ in two-dimensional $\mathcal{N}=(2,2)$ SCFT's with $c=9$, obtained by combining the worldsheet with spacetime arguments, was used to prove that the conformal manifold of such SCFT's factorizes (1.1). The result in [8] follows from the vanishing of the mixed chiral and twisted chiral curvature components, which implies that the holonomy group is the direct product of two commuting subgroups, which in turn implies factorization (1.1). In this section we establish the following result about any $\mathcal{N}=(2,2)$ SCFT by investigating the curvature on its conformal manifold explicitly: the conformal manifold of an $\mathcal{N}=(2,2)$ SCFT does not factorize if and only if the SCFT has a conserved current with R-charges $(2,0)$.

We now proceed to compute the mixed components of the Riemann tensor of $\mathcal{M}$ using CFT techniques, in the spirit of $[3,19,41,42]$. The tangent bundle $T \mathcal{M}$ comes equipped with a metric compatible connection, whose curvature we would like to find. The curvature of this connection is determined by a certain four-point function of the exactly marginal operators, which we denote by $\mathcal{U}_{i}$. The formula for the curvature can be written as $[42]^{20}$

$$
R_{i j k l}=-R V \int \frac{d^{2} y}{\pi} \log \left(y^{++} y^{--}\right)\left\langle\mathcal{U}_{i}(0) \mathcal{U}_{k}(y) \mathcal{U}_{l}(1) \mathcal{U}_{j}(\infty)\right\rangle_{c} .
$$

The symbol 'RV' stands for the prescription where we cut out small discs around the fixed operators and remove the power-law divergent terms. ${ }^{21}$ The subscript $c$ stands for the

\footnotetext{
${ }^{20}$ Note that our definition of the curvature includes an overall normalization of 4 compared to that of [42]. This is the convention in which the special geometry relation takes the standard form. In addition, we define as usual $O(\infty)=\lim _{x \rightarrow \infty} x^{2 \Delta_{O}} O(x)$ while keeping all the other insertions fixed.

${ }^{21}$ The energy-momentum tensor would appear in the OPE with a $1 / y^{2}$ singularity. However, it has an angular dependence and thus this singularity vanishes upon integrating over the angles. We recall that dimension $(1,1)$ operators do not appear in the OPE since a nonzero OPE coefficient would lead to a beta function.
} 
connected correlator, which is defined by subtracting the three possible disconnected contributions to the four-point function. As shown in [41], an explicit formula for the curvature can be given in terms of the CFT data: spectrum of operators and OPE coefficients.

Extending the $\mathcal{N}=(2,2)$ superconformal Ward identities first introduced in [8] and further exploited in [19] we can show that the only non-trivial mixed chiral and twisted chiral moduli four-point function that needs to be studied is

$$
\left\langle F_{i}(x) \bar{F}_{\bar{j}}(y) \tilde{F}_{\tilde{k}}(z) \overline{\tilde{F}}_{\overline{\tilde{\ell}}}(w)\right\rangle,
$$

where we have denoted the exactly marginal operators constructed from operators in the chiral and twisted chiral ring by

$$
F_{i}=\frac{1}{2} \int d \theta^{+} d \theta^{-} \mathcal{O}_{i}, \quad \tilde{F}_{\tilde{i}}=\frac{1}{2} \int d \theta^{+} d \bar{\theta}^{-} \tilde{\mathcal{O}}_{\tilde{i}} .
$$

Using the superconformal Ward identities introduced in [8], the four-point function of interest (4.2) can be expressed in terms of the four-point function of chiral and twisted chiral operators of dimension $\left(\frac{1}{2}, \frac{1}{2}\right)$

$$
\begin{aligned}
& \left\langle F_{i}(x) \bar{F}_{\bar{j}}(y) \tilde{F}_{\tilde{k}}(z) \overline{\tilde{F}}_{\tilde{\tilde{\ell}}}(w)\right\rangle \\
& =\partial_{y^{++}} \partial_{y^{--}} \partial_{z^{--}} \partial_{w^{++}}\left[\frac{y^{++}-w^{++}}{z^{++}-x^{++}} \frac{y^{--}-z^{--}}{x^{--}-w^{--}}\left\langle\mathcal{O}_{i}(x) \overline{\mathcal{O}}_{\bar{j}}(y) \tilde{\mathcal{O}}_{\tilde{k}}(z) \overline{\tilde{\mathcal{O}}}_{\tilde{\ell}}(w)\right\rangle\right] .
\end{aligned}
$$

We are now ready to compute the curvature using (4.1). It follows from (4.4) that the independent components of the Riemann curvature tensor are $R_{i \tilde{k} \tilde{\ell} \bar{j}}$ and $R_{i \tilde{\tilde{\ell}} \tilde{k} \bar{j}}$. The connected component prescription in (4.1) can be extended to both sides of (4.4), and therefore, by pulling the operator located at $x$ to infinity we arrive at

$$
\begin{aligned}
& \left\langle F_{i}(\infty) \bar{F}_{\bar{j}}(y) \tilde{F}_{\tilde{k}}(z) \overline{\tilde{F}}_{\overline{\tilde{\ell}}}(w)\right\rangle_{c} \\
& =\partial_{y^{++}} \partial_{y^{--}} \partial_{z^{--}} \partial_{w^{++}}\left[\left(y^{++}-w^{++}\right)\left(y^{--}-z^{--}\right)\left\langle\mathcal{O}_{i}(\infty) \overline{\mathcal{O}}_{\bar{j}}(y) \tilde{\mathcal{O}}_{\tilde{k}}(z) \overline{\tilde{\mathcal{O}}}_{\bar{\ell}^{(}}(w)\right\rangle_{c}\right] .
\end{aligned}
$$

Integrating by parts in (4.1) and remembering that we are integrating over the complex plane with disks around the punctures removed, the answer reduces to contour integrals around the punctures. In order to get a non-zero contribution to the curvature, the function

$$
\left.g(y) \equiv \partial_{z^{--}} \partial_{w^{++}}\left[\left(y^{++}-w^{++}\right)\left(y^{--}-z^{--}\right)\left\langle\mathcal{O}_{i}(\infty) \overline{\mathcal{O}}_{\bar{j}}(y) \tilde{\mathcal{O}}_{\tilde{k}}(z) \overline{\mathcal{O}}_{\tilde{\ell}}(w)\right\rangle_{c}\right]\right|_{z=0, w=1}
$$

must either have a constant piece at $y=0$ or have a simple pole at $y=1 .^{22}$ Let us analyze $g(y)$ near $y=1$. This leads us to consider the OPE studied in section 2

$$
\overline{\mathcal{O}}_{\bar{j}}(y) \overline{\tilde{\mathcal{O}}}_{\overline{\hat{\ell}}}(w) \sim \frac{C_{\bar{j} \bar{\ell}}}{y^{--}-w^{--}} \bar{J}_{++}(w),
$$

\footnotetext{
${ }^{22}$ More singular terms are removed by the prescription in (4.1). One can verify that there is no contribution from infinity.
} 
where $\bar{J}_{++}$has R-charges $(-2,0)$. Using

$$
\left\langle\bar{J}_{++}(w) \tilde{\mathcal{O}}_{\tilde{k}}(z) \mathcal{O}_{i}(\infty)\right\rangle=\frac{C_{i \tilde{k}}}{w^{++}-z^{++}},
$$

we find that indeed near $y=1$ the function $g(y)$ has a simple pole

$$
g(y) \sim \frac{y^{++}}{y^{--}-1} .
$$

A very similar analysis of the behavior of the function near $y=0$, where now the relevant OPE is

$$
\mathcal{O}_{\bar{j}}(y) \tilde{\mathcal{O}}_{\tilde{k}}(z) \sim \frac{C_{\bar{j} \tilde{k}}}{y^{++}-z^{++}} \bar{J}_{--}(z),
$$

demonstrates that there is no contribution to the curvature from the contour integral around $y=0$.

In conclusion, we have shown that there is a non-trivial component of the mixed curvature tensor between chiral and twisted chiral moduli in an $\mathcal{N}=(2,2)$ SCFT if and only if the SCFT has a current with R-charges $(2,0)$. In such a SCFT, the Riemann curvature is given by

$$
R_{i \tilde{\bar{k}} \tilde{\tilde{\ell}} \bar{j}} \sim C_{\bar{j} \overline{\tilde{\ell}}} C_{i \tilde{k}}, \quad R_{i \overline{\tilde{\ell}} \tilde{k} \bar{j}} \sim C_{\bar{j} \tilde{k}} C_{i \tilde{\ell}}
$$

and the conformal manifold $\mathcal{M}$ no longer factorizes.

\section{$5 \mathcal{N}=(4,4)$ conformal manifolds}

The small $\mathcal{N}=4$ superconformal algebra, which has an $\mathrm{SU}(2)_{R}$ R-symmetry (see e.g. [19]), is an important example of extended supersymmetry. In this section, we use the formula (4.1) to compute the curvature on the conformal manifold of $\mathcal{N}=(4,4)$ theories and give a purely field-theoretic derivation that the local geometry of the coset is (1.4). We also study the bundle of $\mathcal{N}=4$ supercurrents over the conformal manifold and show that there is no consistent choice of an $\mathcal{N}=2$ subalgebra even over a local coordinate patch. This gives a geometric perspective on our shortening anomaly.

Let us introduce some notation first: the left-moving $\mathcal{N}=4$ supercurrents are denoted by $S_{+++}^{\alpha A}$, and the $\mathrm{SU}(2)_{R}$ currents by $J_{++}^{(\alpha \beta)}$, where $\alpha$ and $A$ are doublet indices for the R-symmetry $\mathrm{SU}(2)_{R}$ and the outer automorphism $\mathrm{SU}(2)_{\text {out }}$ of the $\mathcal{N}=4$ superconformal algebra, respectively.

We use the convention $\epsilon^{12}=\epsilon_{21}=1$ for the invariant tensors $\epsilon_{\alpha \beta}, \epsilon_{A B}$ and their inverses. For the right moving sector, we use dotted indices. We denote the weight $\left(\frac{1}{2}, \frac{1}{2}\right)$ BPS primaries by $\mathcal{O}_{i \alpha \dot{\alpha}}$, where $i=1, \ldots, n$. Their weight $(1,1)$ descendants

$$
F_{i}^{A \dot{A}}=\frac{1}{8}\left\{Q_{+}^{\alpha A},\left[Q_{-}^{\dot{\alpha} \dot{A}}, \mathcal{O}_{a \alpha \dot{\alpha}}\right]\right\}
$$

are exactly marginal operators which preserve the $\mathcal{N}=(4,4)$ superconformal symmetry $[2,8,19]$. These operators span the conformal manifold $\mathcal{M}$ of $\mathcal{N}=(4,4)$ SCFT's. Their two-point functions are

$$
\left\langle\mathcal{O}_{i \alpha \dot{\alpha}}(x) \mathcal{O}_{j \beta \dot{\beta}}(y)\right\rangle=\frac{\eta_{i j} \epsilon_{\alpha \beta} \epsilon_{\dot{\alpha} \dot{\beta}}}{(x-y)^{2}}
$$


and

$$
\left\langle F_{i A \dot{A}}(x) F_{j B \dot{B}}(y)\right\rangle=\frac{\eta_{i j} \epsilon_{A B} \epsilon_{\dot{A} \dot{B}}}{(x-y)^{4}},
$$

where $\eta_{i j}$ is the Zamolodchikov metric.

\subsection{Riemannian curvature}

In the $\mathcal{N}=4$ notation above, the formula (4.1) for the Riemann curvature is expressed as

$$
R_{i A \dot{A} ; j B \dot{B} ; k C \dot{C} ; \ell D \dot{D}}=-R V \int \frac{d^{2} y}{\pi} \log \left|y^{2}\right|\left\langle F_{i A \dot{A}}(0) F_{j B \dot{B}}(y) F_{k C \dot{C}}(1) F_{\ell D \dot{D}}(\infty)\right\rangle_{c} .
$$

By choosing an $\mathcal{N}=2$ subalgebra generated by $S_{+++}^{11}$ and $S_{+++}^{22}$, marginal operators for (anti-)chiral multiples are $F_{i 11}\left(F_{i 2 \dot{2}}\right)$, while those for (anti-)twisted chiral multiples are $F_{i 1 \dot{2}}\left(F_{i 2 \dot{1}}\right)$.

As shown in the previous section, there are non-zero curvature components in mixed chiral and twisted chiral directions, which in the $\mathcal{N}=4$ notation can be expressed as

$$
R_{i 1 \dot{1} ; j 1 \dot{2} ; k 2 \dot{1} ; \ell 2 \dot{2}}=R_{i 1 \dot{1} ; j 2 \dot{1} ; k 1 \dot{2} ; \ell 2 \dot{2}}=-\frac{1}{k} \eta_{i j} \eta_{k \ell},
$$

where $k$ in the normalization factor is related to the Virasoro central charge by $c=6 k$. By using the Bianchi identity, we can also determine ${ }^{23}$

$$
R_{i 2 \dot{1} ; j 1 \dot{2} ; k 1 \dot{i} ; \ell 2 \dot{2}}=-\frac{1}{k}\left(\eta_{i \ell} \eta_{j k}-\eta_{i k} \eta_{j \ell}\right) .
$$

On the other hand, the curvatures in the purely chiral directions are controlled by the special geometry relation for $c=9$ and its generalization for other $c[10,12,13,43]$, which we re-derive in appendix B using the formula (4.1). In the $\mathcal{N}=4$ notation, it takes the form

$$
R_{i 1 ; ; j 2 \dot{2} ; k 1 \dot{1} ; \ell 2 \dot{2}}=\eta_{i j} \eta_{k \ell}+\eta_{i \ell} \eta_{j k}-C_{i k}{ }^{I} C_{j \ell}{ }^{J} g_{I J}
$$

where $C_{i k}^{J}$ are the chiral ring coefficients.

In appendix $\mathrm{D}$, we use the $\mathcal{N}=(4,4)$ superconformal symmetry to find a relation between the curvature components (5.5), (5.6), and (5.7)

$$
R_{i 1 \dot{1} ; j 2 \dot{2} ; k 1 \dot{1} ; \ell 2 \dot{2}}+R_{i 2 \dot{1} ; j 12 ; k 1 \dot{i} ; \ell 2 \dot{2}}+R_{i 2 \dot{1} ; j 2 \dot{2} ; k 1 \dot{1} ; \ell 1 \dot{2}}=0 .
$$

This allows us to determine

$$
R_{i 1 \dot{1} ; j 2 \dot{2} ; k 1 \dot{1} ; \ell 2 \dot{2}}=\frac{1}{k}\left(\eta_{i \ell} \eta_{j k}-\eta_{i k} \eta_{j \ell}+\eta_{i j} \eta_{k \ell}\right) .
$$

Comparing this with (5.7), we obtain as a by-product the following constraint on the chiral ring of any $\mathcal{N}=(4,4)$ SCFT

$$
C_{i k}^{I} C_{j \ell}^{J} g_{I J}=\left(1-\frac{1}{k}\right)\left(\eta_{i j} \eta_{k \ell}+\eta_{i \ell} \eta_{k j}\right)+\frac{1}{k} \eta_{i k} \eta_{j \ell} .
$$

\footnotetext{
${ }^{23}$ The Bianchi identity $R_{i[j k \ell]}=0$ follows from the crossing symmetry of the four-point function [42].
} 
For an $\mathcal{N}=(4,4)$ SCFT realized by a sigma model on a hyper-Kähler manifold $M$, it would be nice to verify that the cohomology ring of $M$ satisfies the constraint (5.10). ${ }^{24}$ Combining these results, the full Riemannian curvature is

$$
\begin{aligned}
& R_{i A \dot{A} ; j B \dot{B} ; k C \dot{C} ; \ell D \dot{D}}=-\frac{1}{k}\left(\eta_{i k} \eta_{j \ell}-\eta_{i \ell} \eta_{j k}\right) \epsilon_{A B} \epsilon_{C D} \epsilon_{\dot{A} \dot{B}} \epsilon_{\dot{C} \dot{D}} \\
& \quad-\frac{1}{2 k} \eta_{i j} \eta_{k \ell}\left(\left(\epsilon_{\dot{A} \dot{C}} \epsilon_{\dot{B} \dot{D}}+\epsilon_{\dot{A} \dot{D}} \epsilon_{\dot{B} \dot{C}}\right) \epsilon_{A B} \epsilon_{C D}+\left(\epsilon_{A C} \epsilon_{B D}+\epsilon_{A D} \epsilon_{B C}\right) \epsilon_{\dot{A} \dot{B}} \epsilon_{\dot{C} \dot{D}}\right) .
\end{aligned}
$$

This implies that the conformal manifold of an $\mathcal{N}=(4,4)$ SCFT is locally the coset manifold (1.4)

$$
\mathcal{M}=\frac{O(4, n)}{O(4) \times O(n)}
$$

\subsection{Supercurrent bundle}

Let us turn to the curvature of the bundle of the $\mathcal{N}=4$ supercurrents $S_{+++}^{\alpha A},{ }^{25}$

$$
R_{i C \dot{C} j D \dot{D} \alpha A \beta B}=-\frac{1}{4 k} R V \int \frac{d^{2} y}{\pi} \log \left(y^{++} y^{--}\right)\left\langle S_{\alpha A+++}(0) F_{i C \dot{C}}(y) F_{j D \dot{D}}(1) S_{\beta B+++}(\infty)\right\rangle_{c} .
$$

In appendix $\mathrm{C}$, we determine the connected four-point function using $\mathcal{N}=(4,4)$ Ward identities to be

$$
\left\langle S_{+++}^{\alpha A}(0) F_{i}^{C \dot{C}}(y) F_{j}^{D \dot{D}}(1) S_{+++}^{\beta B}(\infty)\right\rangle_{c}=-2 \epsilon^{\alpha \beta} \epsilon^{\dot{C} \dot{D}} \eta_{i j} \partial_{y^{++}} \partial_{y^{--}}\left(\frac{\epsilon^{A C} \epsilon^{B D}}{y(\bar{y}-1)}+\frac{\epsilon^{A D} \epsilon^{B C} y}{\bar{y}-1}\right) .
$$

The $y$-integral in (5.13) can then be performed

$$
R_{i C \dot{C} ; j D \dot{D} ; \alpha A ; \beta B}=-\frac{1}{2 k} \eta_{i j} \epsilon_{\dot{C} \dot{D}} \epsilon_{\alpha \beta}\left(\epsilon_{A C} \epsilon_{B D}+\epsilon_{A D} \epsilon_{B C}\right),
$$

and

$$
R_{i C \dot{C} ; j D \dot{D} ; \dot{\alpha} \dot{A} ; \dot{\beta} \dot{B}}=-\frac{1}{2 k} \eta_{i j} \epsilon_{C D} \epsilon_{\dot{\alpha} \dot{\beta}}\left(\epsilon_{\dot{A} \dot{C}} \epsilon_{\dot{B} \dot{D}}+\epsilon_{\dot{A} \dot{D}} \epsilon_{\dot{B} \dot{C}}\right) .
$$

The nontrivial $\mathrm{SU}(2)_{\text {out }} \times \mathrm{SU}(2)_{\text {out }}$ holonomies shown in these curvatures means that it is not possible to choose an $\mathcal{N}=2$ subalgebra consistently, even on a local patch of $\mathcal{M}$.

Since the tangent bundle $T \mathcal{M}$ is a tensor product of the left and right supercurrent bundles and the bundle of weight $\left(\frac{1}{2}, \frac{1}{2}\right)$ chiral primaries, the curvature tensor for the supercurrents computed here can be combined with the curvatures for the chiral primaries computed in [19] to reproduce the Riemann curvature (5.11) on $\mathcal{M}$.

Although $\mathcal{M}$ is not Kähler, it has Kähler sub-manifolds. In fact, the maximal Kähler sub-manifold of a quaternionic-Kähler manifold is middle-dimensional [44]. In our case, the maximal Kähler submanifold of $\mathcal{M}$ is locally

$$
\mathcal{S}=\frac{O(2, n)}{O(2) \times O(n)}
$$

\footnotetext{
${ }^{24} \mathrm{~A}$ curious observation is that (5.10) implies that there is a uniform bound on chiral ring coefficients (squared) associated with the R-charge $(1,1)$ chiral primaries. The bound takes the schematic form $C^{2}<$ $2-1 / k$. Note that this is very different from the $\mathcal{N}=(2,2)$ case, where the chiral ring coefficients can blow up, say, as at the conifold point.

${ }^{25}$ The $k$ dependent normalization is due to the two-point function $\left\langle S_{+++}^{\alpha A}(0) S_{+++}^{\beta B}(\infty)\right\rangle=4 k \epsilon^{\alpha \beta} \epsilon^{A B}$.
} 
If we only turn on the marginal couplings associated to chiral multiplets and explore that sub-manifold of $\mathcal{M}$, then there is no shortening anomaly, and the argument of [14] leading to Kählerity applies. In $\mathcal{N}=(4,4)$ SCFTs, the subspace spanned by the marginal couplings associated to chiral or twisted chiral operators corresponds to the sub-manifold (5.17) .

\section{Acknowledgments}

We thank Kevin Costello, David Morrison, Kyriakos Papadodimas, Ronen Plesser, Adam Schwimmer, Stefan Theisen, and Edward Witten for useful discussions. J.G.'s research was supported in part by the Perimeter Institute for Theoretical Physics. Research at Perimeter Institute is supported by the Government of Canada through Industry Canada and by the Province of Ontario through the Ministry of Research and Innovation. Z.K. is supported in part by an Israel Science Foundation center for excellence grant and by the I-CORE program of the Planning and Budgeting Committee and the Israel Science Foundation (grant number 1937/12). Z.K. is also supported by the ERC STG grant 335182 and by the United States-Israel BSF grant 2010/629. H.O. is supported in part by U.S. Department of Energy grant DE-SC0011632, by the Simons Investigator Award, by the World Premier International Research Center Initiative, MEXT, Japan, by JSPS Grantin-Aid for Scientific Research C-26400240, and by JSPS Grant-in-Aid for Scientific Research on Innovative Areas 15H05895. N.S. was supported in part by DOE grant DE-SC0009988. Y.W. was supported by the NSF grant PHY-1620059 and by the Simons Foundation Grant \#488653. H.O. thanks the hospitality of the Institute for Advanced Study and Harvard University, where he spent his sabbatical in 2015 - 2016, and of the Aspen Center for Physics, which is supported by the National Science Foundation grant PHY-1066293. N.S. thanks the hospitality of the Weizmann Institute of Science during the completion of this work.

\section{A The Wess-Zumino perspective}

Anomalies arise when we have some operator that should satisfy an operator equation, e.g. $\partial^{\mu} j_{\mu}=0$ or $T_{\mu}^{\mu}=0$, but then one finds that such an equation is only correct at separated points while at coincident points there are various contact terms. The essence is to show that these contact terms cannot be removed by redefining the scheme. Indeed, scheme redefinitions change the theory by various contact terms and so we need to demonstrate that the anomaly is invariant under scheme redefinitions. A convenient way to establish it is to introduce background fields for the various operators. Then scheme redefinitions correspond to adding new local terms to the action, which depend on these background fields and also, possibly, on the operators in the theory.

We would like to examine the operator $\left[\bar{Q}_{+}, \mathcal{O} \mid\right]$, which is normally zero if $\mathcal{O}$ is chiral. To this end we couple a background field to this operator. A standard procedure is to couple the superfield $\mathcal{O}$ to a background field in the superpotential but then we do not have a source for the redundant operator. Therefore we will couple $\mathcal{O}$ to a source in the 
Kähler potential. We add a corresponding term for a twisted chiral superfield $\mathcal{O}$ :

$$
\int d^{4} \theta(A \mathcal{O}+B \tilde{\mathcal{O}}+\text { c.c. })
$$

Now we imagine computing the partition function $Z[A, B]$ (with $A, B$ superfields). It is useful to tabulate their charges

$$
\begin{aligned}
& \mathrm{U}(1)_{+} \times \mathrm{U}(1)_{-} \text {Dimension } \\
& \begin{array}{llll}
A & -1 & -1 & (-1 / 2,-1 / 2)
\end{array} \\
& B \quad-1 \quad 1 \quad(-1 / 2,-1 / 2)
\end{aligned}
$$

The standard expectation is that the partition function would not actually depend on most of the components in $A, B$ due the fact that $\left[\bar{Q}_{ \pm}, \mathcal{O} \mid\right]=0$ and $\left[\bar{Q}_{+}, \tilde{\mathcal{O}} \mid\right]=\left[Q_{-}, \tilde{\mathcal{O}} \mid\right]=$ $0 .{ }^{26}$ So the standard expectation is that

$$
\begin{aligned}
& Z[A, B]=Z\left[A+\bar{D}_{+} \chi_{-}+\bar{D}_{-} \chi_{+}, B\right] \\
& Z[A, B]=Z\left[A, B+\bar{D}_{+} \psi_{-}+D_{-} \psi_{+}\right]
\end{aligned}
$$

for arbitrary $\chi_{ \pm}, \psi_{ \pm}$. This should be viewed, for example, in analogy with $\delta_{\sigma} Z\left[g_{\mu \nu}\right]=0$ for the conformal anomaly case. What we would like to test is whether we can respect (A.3) and (A.4) while preserving $\mathcal{N}=(2,2)$ supersymmetry. In other words, we want to see whether the shortening of the background multiplets is consistent with supersymmetry.

The general principles that we reviewed above tell us that for infinitesimal $\chi_{ \pm}, \psi_{ \pm}$, $\delta_{\chi_{ \pm}, \psi_{ \pm}} Z[A, B]$ should be a local functional of the sources and operators in the theory i.e.

$$
\delta_{\chi_{ \pm}, \psi_{ \pm}} \log Z[A, B]=\int \chi_{-} L_{l o c a l}^{-}+\cdots,
$$

with $L_{\text {local }}$ some local function of the couplings and operators. The right hand side in (A.5) is restricted by demanding that it is supersymmetric and also by demanding that it obeys the Wess-Zumino consistency conditions [45].

Let us assume that the partition function is invariant under the $\psi_{ \pm}$transformations, namely, $\tilde{\mathcal{O}}$ obeys the twisted chiral shortening conditions at both separated and coincident points. We can then write the variation under $\chi_{-}$as follows (the formula for the variation under $\chi_{+}$is analogous)

$$
\delta_{\chi_{-}} \log Z[A, B]=\kappa \int d^{4} \theta \bar{D}_{+} D_{-} B \bar{D}_{-} \chi_{-} \mathcal{J}_{++},
$$

with $\kappa$ some constant. Equation (A.6) respects supersymmetry (because it is a $\int d^{4} \theta$ integral), and it is consistent with the R-symmetry $\left(\bar{D}_{+} D_{-} B\right.$ carries R-charges $(0,0)$ and $\chi_{\text {- }}$ carries R-charges $(-2,-1)$ and therefore $\bar{D}_{-} \chi_{-}$carries $(-2,0)$ and thus it exactly cancels the $R$-charge of $\mathcal{J}_{++}$). Furthermore, (A.6) obeys the Wess-Zumino consistency

\footnotetext{
${ }^{26}$ This standard expectation follows from the fact that these conditions hold "off shell," namely there is a regularization where this is true. Technically, it means that there are no cohomologically nontrivial contact terms in correlation functions of these redundant operators.
} 
condition since $\bar{D}_{+} D_{-} B$ is invariant under $B \rightarrow B+\bar{D}_{+} \psi_{-}+D_{-} \psi_{+}$. Therefore, (A.6) does not violate the fact that the partition function is postulated to be invariant under $\psi_{ \pm}$ transformations.

We now proceed to prove that (A.6) is cohomologically non-trivial. If (A.6) were cohomologically trivial then one could add a local term to $\log Z[A, B]$ such that the right hand side of (A.6) would vanish while retaining supersymmetry and invariance under $\psi_{ \pm}$ transformations. It is clear (by integration by parts and using (2.7)) that the $\chi_{-}$-variation of the local term

$$
\int d^{4} \theta A \bar{D}_{-} D_{-} B \mathcal{J}_{++}
$$

could cancel the right hand side of (A.6). However, (A.7) spoils the invariance of the partition function under $\psi_{ \pm}$transformations. One can easily verify that indeed the right hand side of (A.6) is physical as long as we insist on supersymmetry and invariance under $\psi_{ \pm}$transformations.

To summarize let us make some comments

- 1. Suppose we always insist on preserving $\mathcal{N}=(2,2)$ supersymmetry. Then, if $\kappa$ in (A.6) is nonzero, then it turns out that we may not be able to respect both (A.3) and (A.4). In other words, we cannot embed the coupling constants of chiral and twisted chiral operators into short multiplets. At least some of the couplings have to be in longer multiplets.

- 2. We can view the $\chi$ and $\psi$ transformations as analogous to $\mathrm{U}(1)_{A}$ and $\mathrm{U}(1)_{V}$ transformations in $2 d$ electrodynamics. If we preserve one we must give up on the other, but choosing which one to preserve is at our discretion. Therefore, the situation is very similar to the way the usual chiral 't Hooft anomalies arise [39].

Note that from equation (A.6) we can immediately write the anomaly in operatorial formalism. This is because $\chi_{-}$couples to $\bar{D}_{+} \mathcal{O}$ and so we find

$$
\bar{D}_{+} \mathcal{O} \sim \kappa\left(\bar{D}_{-} \bar{D}_{+} D_{-} B\right) \mathcal{J}_{++} .
$$

But since our partition function is invariant under $\psi_{ \pm}$transformations and hence depends only on $\tilde{\lambda}=\bar{D}_{+} D_{-} B$, which is a standard twisted chiral background field, we can also write

$$
\bar{D}_{+} \mathcal{O} \sim \kappa \bar{D}_{-} \tilde{\lambda} \mathcal{J}_{++}
$$

Hence in a "fermionic background" for the twisted chiral coupling, the operator $\mathcal{O}$ ceases to be chiral.

It is now straightforward to make contact with the analysis in the bulk of the paper. Our discussion in this appendix has shown that there may be an anomaly with coefficient $\kappa$ and that it would manifest itself as (A.9). Comparing with (3.12) we thus see that this coefficient is nonzero whenever the OPE coefficient in (3.9) is nonzero. Furthermore, the analysis in this appendix sheds light on the choices we could make in (3.11). Indeed, we could have chosen whether to postulate that the partition function preserves (A.3), (A.4), or none of the two. As in all cases with anomalies, these various choices are related to 
each other by adding counter-terms to the action, e.g. the one we discussed in (A.7). By choosing the coefficient of this counter-term carefully, we can change the scheme from the one where $\psi$ transformations are obeyed to the one where $\chi$ transformations are obeyed.

\section{B Special geometry relation}

It is well known that for an $\mathcal{N}=(2,2) \mathrm{SCFT}$, the Riemannian curvature for marginal deformations generated by chiral primary fields is determined in terms of the Zamolodchikov metric and the chiral ring coefficients $[10,12,13,43]$. For $c=9$, this is known as the special geometry relation. Below we will give a simple derivation of this relation using (4.1) (our derivation is valid for any central charge $c$ ).

Following [8], we can use $\mathcal{N}=2$ Ward identities to express the integrand of (4.1) as a total derivative,

$$
\begin{aligned}
& \left\langle F_{i}(1) \bar{F}_{\bar{j}}(y) F_{k}(\infty) \bar{F}_{\bar{\ell}}(0)\right\rangle_{c} \\
= & \left.\partial_{y^{++}} \partial_{y^{--}} \partial_{w^{++}} \partial_{w^{--}}\left(\left(y^{++}-w^{++}\right)\left(y^{--}-w^{--}\right)\left\langle\mathcal{O}_{i}(1) \overline{\mathcal{O}}_{\bar{j}}(y) \mathcal{O}_{k}(\infty) \overline{\mathcal{O}}_{\bar{\ell}}(w)\right\rangle_{c}\right)\right|_{w=0} .
\end{aligned}
$$

The curvature is then computed by integration by parts, with non-vanishing contributions coming from the origin $(y=0) .{ }^{27}$ Therefore, we need to study the order-one terms in the $\left.\operatorname{limit}_{\lim _{y \rightarrow 0}} \partial_{w^{++}} \partial_{w^{--}}\left(\left(y^{++}-w^{++}\right)\left(y^{--}-w^{--}\right)\left\langle\mathcal{O}_{i}(1) \overline{\mathcal{O}}_{\bar{j}}(y) \mathcal{O}_{k}(\infty) \overline{\mathcal{O}}_{\bar{\ell}}(w)\right\rangle_{c}\right)\right|_{w=0}$. We can act with the derivatives on the prefactor $|y-w|^{2}$ and thus reduce the problem to studying the $y \rightarrow 0$ limit of $\left\langle\mathcal{O}_{i}(1) \overline{\mathcal{O}}_{\bar{j}}(y) \mathcal{O}_{k}(\infty) \overline{\mathcal{O}}_{\bar{\ell}}(0)\right\rangle_{c}$. There is a contribution from the unit operator in the $t$ and $u$ channel as well as a contribution from the $(2,2)$ chiral primaries,

$$
R_{k \bar{\ell} i \bar{j}}=-\lim _{y \rightarrow 0}\left\langle\mathcal{O}_{i}(1) \overline{\mathcal{O}}_{\bar{j}}(y) \mathcal{O}_{k}(\infty) \overline{\mathcal{O}}_{\bar{\ell}}(0)\right\rangle_{c}=g_{i \bar{j}} g_{k \bar{\ell}}+g_{i \bar{\ell}} g_{k \bar{j}}-\bar{C}_{\bar{j} \bar{\ell}}{ }^{\bar{J}} g_{I \bar{J}} C_{i k}{ }^{I},
$$

where $g_{I \bar{J}}$ is the metric associated with the R-charge $(2,2)$ chiral primaries.

\section{Four-point function involving supercurrents}

Let us focus on the connected four-point function

$$
\left\langle S_{+++}^{\alpha A}(x) F_{i}^{C \dot{C}}(y) F_{j}^{D \dot{D}}(z) S_{+++}^{\beta B}(w)\right\rangle_{c} .
$$

Since the four-point function is holomorphic in $z$ and $w$, it is determined completely by the poles in the OPE between the supercurrents and other insertions. We first consider the singularities in $x$, and denote the polar terms in $(x-w),(x-y),(x-z)$ by $I_{1}, I_{2}, I_{3}$ respectively.

From the OPE of the $\mathcal{N}=4$ supercurrents,

$$
S_{+++}^{\alpha A}(x) S_{+++}^{\beta B}(w)=\epsilon^{A B}\left(\frac{4 k \epsilon^{\alpha \beta}}{(x-w)^{3}}-\frac{4 \sigma_{i}^{\alpha \beta} J^{i}(w)}{(x-w)^{2}}+\frac{2 \epsilon^{\alpha \beta} T(w)-2 \sigma_{i}^{\alpha \beta} \partial J^{i}(w)}{(x-w)}\right)+\ldots
$$

\footnotetext{
${ }^{27} \mathrm{~A}$ priori, there can be boundary contributions from contours around $y=1$ and $\infty$ as well. However careful analysis of the integrand shows that such contributions are absent for this particular four-point function.
} 
we have

$$
I_{1}=\left\langle\frac{2 \epsilon^{A B} \epsilon^{\alpha \beta} T(w)}{(x-w)} F_{i}^{C \dot{C}}(y) F_{j}^{D \dot{D}}(z)\right\rangle
$$

where we have dropped the disconnected pieces and also the terms involving the $\mathrm{SU}(2)_{R}$ currents since $F_{i}$ are $\mathrm{SU}(2)_{R}$ singlets. It is then easy to obtain using the OPE between $T$ and $F_{i}$

$$
I_{1}=\frac{2 \epsilon^{\alpha \beta} \epsilon^{A B} \epsilon^{C D} \epsilon^{\dot{C} \dot{D}} \eta_{i j}}{(x-w)(w-y)^{2}(w-z)^{2}(\bar{y}-\bar{z})^{2}}
$$

Similarly looking at the OPE between $S_{+++}^{\alpha A}$ and $F_{i}^{C \dot{C}}$

$$
S_{+++}^{\alpha A}(z) F^{B \dot{B}}(w)=\frac{1}{2} \partial_{w^{++}}\left(\frac{\epsilon^{\alpha \beta} \epsilon^{A B} Q_{-}^{\dot{\beta} \dot{B}} \mathcal{O}_{\beta \dot{\beta}}}{z^{++}-w^{++}},\right)+\ldots
$$

we have

$$
I_{2}=\frac{1}{2}\left\langle\partial_{y^{++}}\left(\frac{\epsilon^{\alpha \gamma} \epsilon^{A C} Q_{-}^{\dot{\gamma} \dot{C}} \mathcal{O}_{\gamma \dot{\gamma}}(y)}{x-y}\right) F_{j}^{D \dot{D}}(z) S_{+++}^{\beta B}(w)\right\rangle .
$$

Using again (C.5) and also

$$
S_{+++}^{\alpha A}(z) \mathcal{O}_{\beta \dot{\beta}}(w)=\frac{\delta_{\beta}^{\alpha}}{2\left(z^{++}-w^{++}\right)} Q_{+}^{\gamma A} \mathcal{O}_{\gamma \dot{\beta}}(w)+\ldots
$$

we get

$$
I_{2}=\frac{2 \epsilon^{\alpha \beta} \epsilon^{A C} \epsilon^{B D} \epsilon^{\dot{C}} \dot{D} \eta_{i j}(2 y-x-w)}{(\bar{z}-\bar{y})^{2}(y-w)^{2}(z-w)^{2}(x-y)^{2}}
$$

Finally from the OPE between $S_{+++}^{\alpha A}$ and $F_{j}^{D \dot{D}}$ we get

$$
I_{3}=\frac{1}{2}\left\langle F_{i}^{C \dot{C}}(y) \partial_{z^{++}}\left(\frac{\epsilon^{\alpha \delta} \epsilon^{A D} Q_{-}^{\dot{\delta} \dot{D}} \mathcal{O}_{\delta \dot{\delta}}(z)}{x-z}\right) S_{+++}^{\beta B}(w)\right\rangle
$$

and following the same steps as above we obtain

$$
I_{3}=\frac{2 \epsilon^{\alpha \beta} \epsilon^{A D} \epsilon^{B C} \epsilon^{\dot{C}} \dot{D} \eta_{i j}(w+x-2 z)}{(\bar{z}-\bar{y})^{2}(y-w)^{2}(z-w)^{2}(x-z)^{2}} .
$$

Putting together (C.4), (C.8) and (C.10) while taking the limit $w \rightarrow \infty$, we arrive at

$$
\begin{aligned}
& \left\langle S_{+++}^{\alpha A}(0) F_{i}^{C \dot{C}}(y) F_{j}^{D \dot{D}}(1) S_{+++}^{\beta B}(\infty)\right\rangle_{c} \\
= & 2 \epsilon^{\alpha \beta} \epsilon^{\dot{C} \dot{D}} \eta_{i j}\left(\frac{-\epsilon^{A C} \epsilon^{B D}}{y^{2}(\bar{y}-1)^{2}}+\frac{\epsilon^{A D} \epsilon^{B C}}{(\bar{y}-1)^{2}}\right) \\
= & -2 \epsilon^{\alpha \beta} \epsilon^{\dot{C} \dot{D}} \eta_{i j} \partial_{y^{++}} \partial_{y^{--}}\left(\frac{\epsilon^{A C} \epsilon^{B D}}{y(\bar{y}-1)}+\frac{\epsilon^{A D} \epsilon^{B C} y}{\bar{y}-1}\right) .
\end{aligned}
$$




\section{SU(2) out selection rules}

In this appendix we prove that

$$
\begin{aligned}
& \left\langle F_{i 1 \dot{1}}(0) F_{k 1 \dot{1}}(y) F_{\ell 2 \dot{2}}(1) F_{j 2 \dot{2}}(\infty)\right\rangle+\left\langle F_{i 2 \dot{1}}(0) F_{k 1 \dot{1}}(y) F_{\ell 1 \dot{2}}(1) F_{j 2 \dot{2}}(\infty)\right\rangle \\
& +\left\langle F_{i 2 \dot{1}}(0) F_{k 1 \dot{1}}(y) F_{\ell 2 \dot{2}}(1) F_{j 1 \dot{2}}(\infty)\right\rangle=0,
\end{aligned}
$$

follows from $\mathcal{N}=(4,4)$ superconformal Ward identities. More generally, the four-point function of the exactly marginal operators $\left\langle F_{i A \dot{A}} F_{j B \dot{B}} F_{k C \dot{C}} F_{\ell D \dot{D}}\right\rangle$ respects $\mathrm{SU}(2)_{\text {out }}$ Ward identities. $^{28}$

For notational simplicity, let us focus on the left-moving side of (D.1). Using $\mathcal{N}=4$ Ward identities

$$
\begin{aligned}
& \left\langle Q_{+}^{22} \mathcal{O}_{i}^{1}(x) Q_{+}^{22} \mathcal{O}_{k}^{1}(y) Q_{+}^{11} \mathcal{O}_{\ell}^{2}(z) Q_{+}^{11} \mathcal{O}_{j}^{2}(\infty)\right\rangle \\
= & 2 \partial_{y^{++}}\left\langle Q_{+}^{22} \mathcal{O}_{i}^{1}(x) \mathcal{O}_{k}^{1}(y) \mathcal{O}_{\ell}^{2}(z) Q_{+}^{11} \mathcal{O}_{j}^{2}(\infty)\right\rangle-2 \partial_{x^{++}}\left\langle\mathcal{O}_{i}^{1}(x) Q_{+}^{22} \mathcal{O}_{k}^{1}(y) \mathcal{O}_{\ell}^{2}(z) Q_{+}^{11} \mathcal{O}_{j}^{2}(\infty)\right\rangle, \\
& \left\langle Q_{+}^{21} \mathcal{O}_{i}^{1}(x) Q_{+}^{22} \mathcal{O}_{k}^{1}(y) Q_{+}^{12} \mathcal{O}_{\ell}^{2}(z) Q_{+}^{11} \mathcal{O}_{j}^{2}(\infty)\right\rangle \\
= & 2 \partial_{x^{++}}\left\langle\mathcal{O}_{i}^{1}(x) Q_{+}^{22} \mathcal{O}_{k}^{1}(y) \mathcal{O}_{\ell}^{2}(z) Q_{+}^{11} \mathcal{O}_{j}^{2}(\infty)\right\rangle-\left\langle Q_{+}^{21} \mathcal{O}_{i}^{1}(x) Q_{+}^{22} \mathcal{O}_{k}^{1}(y) \mathcal{O}_{\ell}^{2}(z) \mathcal{O}_{j}^{1}(\infty)\right\rangle,
\end{aligned}
$$

and

$$
\begin{aligned}
& \left\langle Q_{+}^{21} \mathcal{O}_{i}^{1}(x) Q_{+}^{22} \mathcal{O}_{k}^{1}(y) Q_{+}^{11} \mathcal{O}_{\ell}^{2}(z) Q_{+}^{12} \mathcal{O}_{j}^{2}(\infty)\right\rangle \\
= & 2 \partial_{y^{++}}\left\langle Q_{+}^{21} \mathcal{O}_{i}^{1}(x) \mathcal{O}_{k}^{1}(y) \mathcal{O}_{\ell}^{2}(z) Q_{+}^{12} \mathcal{O}_{j}^{2}(\infty)\right\rangle+\left\langle Q_{+}^{21} \mathcal{O}_{i}^{1}(x) Q_{+}^{22} \mathcal{O}_{k}^{1}(y) \mathcal{O}_{\ell}^{2}(z) \mathcal{O}_{j}^{1}(\infty)\right\rangle .
\end{aligned}
$$

Moreover, from (C.5) we can derive

$$
\left\langle Q_{+}^{21} \mathcal{O}_{i}^{1}(x) \mathcal{O}_{k}^{1}(y) \mathcal{O}_{\ell}^{2}(z) Q_{+}^{12} \mathcal{O}_{j}^{2}(\infty)\right\rangle=-\left\langle Q_{+}^{22} \mathcal{O}_{i}^{1}(x) \mathcal{O}_{k}^{1}(y) \mathcal{O}_{\ell}^{2}(z) Q_{+}^{11} \mathcal{O}_{j}^{2}(\infty)\right\rangle
$$

Putting together (D.2), (D.3), (D.4) and (D.5), we obtain

$$
\begin{aligned}
& \left\langle Q_{+}^{22} \mathcal{O}_{i}^{1}(x) Q_{+}^{22} \mathcal{O}_{k}^{1}(y) Q_{+}^{11} \mathcal{O}_{\ell}^{2}(z) Q_{+}^{11} \mathcal{O}_{j}^{2}(\infty)\right\rangle+\left\langle Q_{+}^{21} \mathcal{O}_{i}^{1}(x) Q_{+}^{22} \mathcal{O}_{k}^{1}(y) Q_{+}^{12} \mathcal{O}_{\ell}^{2}(z) Q_{+}^{11} \mathcal{O}_{j}^{2}(\infty)\right\rangle \\
& +\left\langle Q_{+}^{21} \mathcal{O}_{i}^{1}(x) Q_{+}^{22} \mathcal{O}_{k}^{1}(y) Q_{+}^{11} \mathcal{O}_{\ell}^{2}(z) Q_{+}^{12} \mathcal{O}_{j}^{2}(\infty)\right\rangle=0
\end{aligned}
$$

which leads to (D.1).

Open Access. This article is distributed under the terms of the Creative Commons Attribution License (CC-BY 4.0), which permits any use, distribution and reproduction in any medium, provided the original author(s) and source are credited.

\footnotetext{
${ }^{28}$ The $\mathrm{SU}(2)_{\text {out }}$ is the outer automorphism group of the $\mathcal{N}=4$ superconformal algebra, but not a symmetry of the full SCFT. However, the operator spectrum of the theory can still be organized into $\mathrm{SU}(2)_{\text {out }}$ representations by postulating that the $\mathcal{N}=4$ superconformal primaries transform as singlets. Here we see that certain correlation functions respect the $\mathrm{SU}(2)_{\text {out }}$ invariance. This is analogous to the bonus $\mathrm{U}(1)_{Y}$ symmetry in the $4 d \mathcal{N}=4$ super Yang-Mills theory [46]. More recently, the outer automorphism group figured in the study of the K3 SCFT from spacetime arguments in [47].
} 


\section{References}

[1] A.B. Zamolodchikov, Irreversibility of the flux of the renormalization group in a $2 D$ field theory, JETP Lett. 43 (1986) 730 [INSPIRE].

[2] N. Seiberg, Observations on the moduli space of superconformal field theories, Nucl. Phys. B 303 (1988) 286 [INSPIRE].

[3] D. Kutasov, Geometry on the space of conformal field theories and contact terms, Phys. Lett. B 220 (1989) 153 [inSPIRE].

[4] J.L. Cardy, Continuously varying exponents and the value of the central charge, J. Phys. A 20 (1987) L891 [INSPIRE].

[5] M. Bershadsky, C. Vafa and V. Sadov, D strings on D manifolds, Nucl. Phys. B 463 (1996) 398 [hep-th/9510225] [INSPIRE].

[6] S.H. Katz, D.R. Morrison and M.R. Plesser, Enhanced gauge symmetry in type-II string theory, Nucl. Phys. B 477 (1996) 105 [hep-th/9601108] [INSPIRE].

[7] W. Lerche, C. Vafa and N.P. Warner, Chiral rings in $N=2$ superconformal theories, Nucl. Phys. B 324 (1989) 427 [INSPIRE].

[8] L.J. Dixon, V. Kaplunovsky and J. Louis, On effective field theories describing (2,2) vacua of the heterotic string, Nucl. Phys. B 329 (1990) 27 [InSPIRE].

[9] M. Dine and N. Seiberg, Microscopic knowledge from macroscopic physics in string theory, Nucl. Phys. B 301 (1988) 357 [inSPIRE].

[10] A. Strominger, Special geometry, Commun. Math. Phys. 133 (1990) 163 [InSPIRE].

[11] L.J. Dixon, Some World Sheet Properties Of Superstring Compactifications, On Orbifolds And Otherwise, proceedings of the 1987 ICTP Summer Workshop, Trieste, Italy, 29 June -7 August 1987.

[12] S. Cecotti and C. Vafa, Topological antitopological fusion, Nucl. Phys. B 367 (1991) 359 [INSPIRE].

[13] M. Bershadsky, S. Cecotti, H. Ooguri and C. Vafa, Kodaira-Spencer theory of gravity and exact results for quantum string amplitudes, Commun. Math. Phys. 165 (1994) 311 [hep-th/9309140] [INSPIRE].

[14] J. Gomis, P.-S. Hsin, Z. Komargodski, A. Schwimmer, N. Seiberg and S. Theisen, Anomalies, conformal manifolds and spheres, JHEP 03 (2016) 022 [arXiv:1509.08511] [INSPIRE].

[15] N. Seiberg, Naturalness versus supersymmetric nonrenormalization theorems, Phys. Lett. B 318 (1993) 469 [hep-ph/9309335] [INSPIRE].

[16] V. Asnin, On metric geometry of conformal moduli spaces of four-dimensional superconformal theories, JHEP 09 (2010) 012 [arXiv:0912.2529] [INSPIRE].

[17] K.S. Narain, New heterotic string theories in uncompactified dimensions $<10$, Phys. Lett. B 169 (1986) 41 [INSPIRE].

[18] S. Cecotti, $\mathcal{N}=2$ Landau-Ginzburg versus Calabi-Yau $\sigma$-models: Nonperturbative aspects, Int. J. Mod. Phys. A 6 (1991) 1749 [inSPIRE].

[19] J. de Boer, J. Manschot, K. Papadodimas and E. Verlinde, The chiral ring of $A d S_{3} / C F T_{2}$ and the attractor mechanism, JHEP 03 (2009) 030 [arXiv:0809.0507] [INSPIRE]. 
[20] D. Green, Z. Komargodski, N. Seiberg, Y. Tachikawa and B. Wecht, Exactly marginal deformations and global symmetries, JHEP 06 (2010) 106 [arXiv:1005.3546] [INSPIRE].

[21] J. Wess and J. Bagger, Supersymmetry and supergravity, Princeton University Press Princeton, U.S.A. (1992), 259 p.

[22] D.Z. Freedman and A. Van Proeyen, Supergravity, Cambridge University Press (2012).

[23] L. Andrianopoli, R. D'Auria and S. Ferrara, Supersymmetry reduction of $N$ extended supergravities in four-dimensions, JHEP 03 (2002) 025 [hep-th/0110277] [INSPIRE].

[24] E. Witten, Phases of $N=2$ theories in two-dimensions, Nucl. Phys. B 403 (1993) 159 [hep-th/9301042] [INSPIRE].

[25] P.S. Aspinwall, K3 surfaces and string duality, hep-th/9611137 [INSPIRE].

[26] N. Doroud, J. Gomis, B. Le Floch and S. Lee, Exact results in D $=2$ supersymmetric gauge theories, JHEP 05 (2013) 093 [arXiv:1206.2606] [INSPIRE].

[27] F. Benini and S. Cremonesi, Partition functions of $\mathcal{N}=(2,2)$ gauge theories on $S^{2}$ and vortices, Commun. Math. Phys. 334 (2015) 1483 [arXiv:1206.2356] [InSPIRE].

[28] J. Gomis and S. Lee, Exact Kähler potential from gauge theory and mirror symmetry, JHEP 04 (2013) 019 [arXiv: 1210.6022] [INSPIRE].

[29] N. Doroud and J. Gomis, Gauge theory dynamics and Kähler potential for Calabi-Yau complex moduli, JHEP 12 (2013) 099 [arXiv:1309.2305] [INSPIRE].

[30] H. Jockers, V. Kumar, J.M. Lapan, D.R. Morrison and M. Romo, Two-Sphere partition functions and Gromov-Witten invariants, Commun. Math. Phys. 325 (2014) 1139 [arXiv: 1208.6244] [INSPIRE].

[31] E. Gerchkovitz, J. Gomis and Z. Komargodski, Sphere partition functions and the Zamolodchikov metric, JHEP 11 (2014) 001 [arXiv:1405.7271] [INSPIRE].

[32] S. Cecotti and C. Vafa, Topological antitopological fusion, Nucl. Phys. B 367 (1991) 359 [INSPIRE].

[33] H. Osborn, $\mathcal{N}=1$ superconformal symmetry in four-dimensional quantum field theory, Annals Phys. 272 (1999) 243 [hep-th/9808041] [INSPIRE].

[34] M.B. Green and N. Seiberg, Contact interactions in superstring theory, Nucl. Phys. B 299 (1988) 559 [INSPIRE].

[35] J.J. Atick, L.J. Dixon and A. Sen, String calculation of Fayet-Iliopoulos d terms in arbitrary supersymmetric compactifications, Nucl. Phys. B 292 (1987) 109 [InSPIRE].

[36] M. Dine, I. Ichinose and N. Seiberg, F terms and D terms in string theory, Nucl. Phys. B 293 (1987) 253 [INSPIRE].

[37] E. Witten, Superstring perturbation theory revisited, arXiv:1209.5461 [INSPIRE].

[38] M.J. Lighthill, An introduction to Fourier analysis and generalized functions, Cambridge Monographs on Mechanics, Cambridge University Press (1958).

[39] Y. Frishman, A. Schwimmer, T. Banks and S. Yankielowicz, The axial anomaly and the bound state spectrum in confining theories, Nucl. Phys. B 177 (1981) 157 [InSPIRE].

[40] K. Ranganathan, Nearby CFTs in the operator formalism: the role of a connection, Nucl. Phys. B 408 (1993) 180 [hep-th/9210090] [INSPIRE]. 
[41] K. Ranganathan, H. Sonoda and B. Zwiebach, Connections on the state space over conformal field theories, Nucl. Phys. B 414 (1994) 405 [hep-th/9304053] [INSPIRE].

[42] D. Friedan and A. Konechny, Curvature formula for the space of 2-d conformal field theories, JHEP 09 (2012) 113 [arXiv: 1206.1749] [INSPIRE].

[43] M. Baggio, V. Niarchos and K. Papadodimas, $t t^{*}$ equations, localization and exact chiral rings in $4 d \mathcal{N}=2$ SCFTs, JHEP 02 (2015) 122 [arXiv: 1409.4212] [INSPIRE].

[44] D.V. Alekseevsky and S. Marchiafava, A twistor construction of Kähler submanifolds of a quaternionic Kähler manifold, Ann. Mat. Pura Appl. 184 (2005) 53.

[45] J. Wess and B. Zumino, Consequences of anomalous Ward identities, Phys. Lett. B 37 (1971) 95 [INSPIRE].

[46] K.A. Intriligator and W. Skiba, Bonus symmetry and the operator product expansion of $N=4$ Super Yang-Mills, Nucl. Phys. B 559 (1999) 165 [hep-th/9905020] [InSPIRE].

[47] Y.-H. Lin, S.-H. Shao, Y. Wang and X. Yin, Supersymmetry constraints and string theory on K3, JHEP 12 (2015) 142 [arXiv: 1508.07305] [INSPIRE]. 